Supporting Information

\title{
Cycloadditions of Cyclopentadiene and Cycloheptatriene with Tropones: All Endo [6+4] Cycloadditions are Ambimodal
}

\author{
Cooper S. Jamieson, ${ }^{1, \pi}$ Arkajyoti Sengupta, ${ }^{1, \pi}$ and K. N. Houk ${ }^{1, *}$ \\ ${ }^{1}$ Department of Chemistry and Biochemistry, University of California, Los Angeles, CA 90095 \\ I these authors contributed equally. \\ * correspondence should be addressed to: houk@,chem.ucla.edu \\ Table of Contents
}

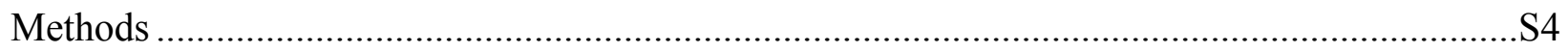

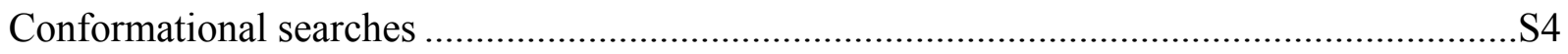

Quantum mechanical geometry optimizations and frequency calculations .............................S5

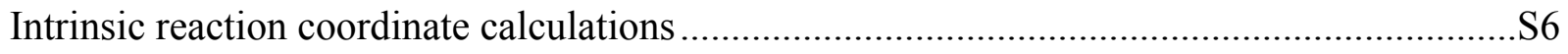

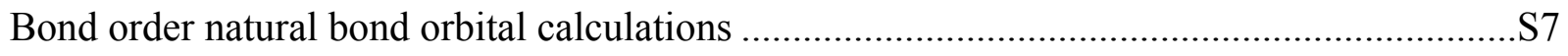

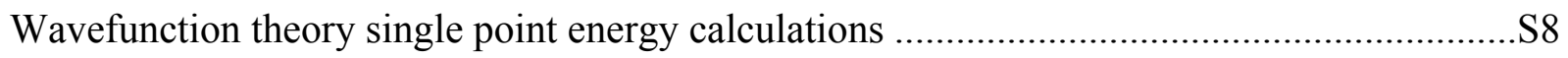

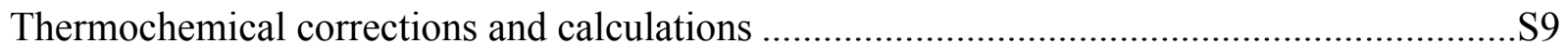

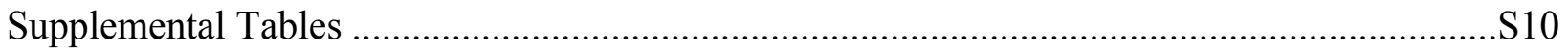

Supplemental Table S1: The calculated bond-order by natural bond orbital (NBO) population for $\mathrm{C}-\mathrm{O}$ bond formation $(\mathrm{O} 8-\mathrm{C} 2$, see Figure 3), ...............................................................

Supplemental Table S2: Energies and bond lengths of transition states with the $\omega$ B97X-D functional and either Ahlrich's def2-TZVP or Pople's 6-31G(d) basis sets. ..........................S11

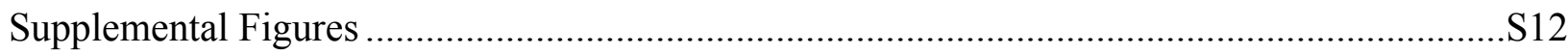

Supplemental Figure S1. Highest-occupied and lowest-unoccupied molecular orbital (HOMO and LUMO) calculations for tropone (1), cyclopentadiene (2), and cycloheptatriene (7)......S12 Supplemental Figure S2. Calculated reactions of cyclopentadiene (2) and tropone (1) at the DLPNO-CCSD(T)/cc-pVQZ// $\omega$ B97X-D/def2-TZVP level of theory ....................................S13

Supplemental Figure S3. Calculated reactions of cycloheptatriene (2) and substituted tropones $(1 \mathrm{a}, 1 \mathrm{~b})$ at the DLPNO-CCSD(T)/cc-pVQZ// $\omega$ B97X-D/def2-TZVP level of theory. ...S14-S15

Supplemental Figure S4. Intrinsic reaction coordinate calculations.......................................S16

Supplemental Figure S5. Quasi-classical reaction dynamics simulations................................S17

Cartesian coordinates of calculated structures ..................................................................... 18

Cyclopentadiene (2) and tropone (1) calculated structures ..................................................S18

1

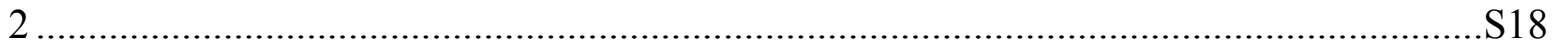

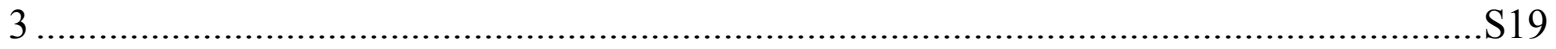




Cycter.




TS-8a




\section{Methods}

\section{Conformational searches}

Conformational searches were conducted using $\mathrm{XTB}^{1}$ and CREST 2.7.1 ${ }^{2}$ on all calculated ground states and transition states. Ground state structures were built or pulled from the CACTUS database and optimized using XTB using a -o loose criteria then reoptimized using -o tight. The XTB optimized structures were then used as an input for a CREST conformational search using the default keywords with -metac

Transition states were located at the $\omega$ B97X-D/def2-svp ${ }^{3,4}$ level of theory and then subjected to constrained conformational searching by freezing the forming bond distances using the following keywords:

-metac -rthr 0.5

Bonds were frozen using the .constrains file with a force constant of 1.0. 


\section{Quantum mechanical geometry optimizations and frequency calculations}

The outputs of conformational searches were optimized using density functional theory paired with an Ahlrichs basis set, $\omega$ B97X-D/def2-TZVP, ${ }^{3-5}$ as implemented in Gaussian 16, revision A.03 sse $4 .^{6}$ This functional was chosen for its ability to calculate geometries of asynchronous Diels-Alder reactions. ${ }^{7}$ Due to the small size of the systems of interest $(\sim<50$ atoms) we could utilize a large triple zeta basis set, which is recommended for use with density functionals. ${ }^{8}$ Structures were characterized as minima or transition states by frequency calculations.

Previous work reported the first tripericyclic reaction and reported calculations using a smaller basis set, 6-31G(d). ${ }^{9}$ There is a minimal geometric difference between the transition states calculated at the def2-TZVP level of theory and the 6-31G(d) level of theory. Please refer to Supplemental Table 2 therein the bond lengths and energies are reported with the Ahlrichs and Pople style basis sets. The differences of forming bond lengths in the transition state due to the basis set are $\sim 0.01 \AA$. 


\section{Intrinsic reaction coordinate calculations}

Intrinsic reaction coordinate calculations were conducted with the following keywords:

irc $=($ lqa, maxpoints $=50$, maxcycle $=200$, calcfc, nogradstop $)$

These calculations were conducted to confirm one of the products that the ambimodal transition states connected.

To see calculation results, please refer to Supplemental Figure S4. 


\section{Bond order natural bond orbital calculations}

Natural orbital analysis of the total density was performed with following keywords:

wb97xd def2tzvp density $=$ current output $=$ wfx pop=NO

Density-Derived Electrostatic and Chemical (DDEC6) calculations on Chargemol version 3.5 using the wavefunction (.wfx) generated from the Gaussian calculations resulted in the bond orders tabulated in Supplemental Table S1. 


\section{Wavefunction theory single point energy calculations}

Single point energetics were calculated using ORCA 4.1.110,11 with the DLPNO-CCSD(T) method $^{12}$ and the cc-pVQZ basis set ${ }^{13}$ with cc-pVQZ/C $\mathrm{C}^{14}$ and def $2 / \mathrm{J}$ auxiliary basis sets ${ }^{15}$ with the manual recommended ZORA and RIJCOSX approximations.

The single point energetics used the following keywords:

! DLPNO-CCSD(T) cc-pvqz cc-pvqz/C Def2/J ZORA RIJCOSX GRIDX4 Grid4 FinalGrid5 TightSCF KDIIS

We were gratified that on 8 cores with access to $7000 \mathrm{mb}$ per core, these jobs took $\sim 1-5 \mathrm{~h}$ to terminate. 


\section{Thermochemical corrections and calculations}

Energetics were calculated using GoodVibes 3.0.0 and 3.0.1 ${ }^{16}$ implementing vibrational scaling $(0.975),{ }^{17}$ quasi-harmonic enthalpy ${ }^{18}$ and entropy corrections ${ }^{19}$ with the following keywords:

$-c 1-q--s p c S P$ 


\section{Supplemental Tables}

Supplemental Table S1: The calculated bond-order by natural bond orbital (NBO) population for $\mathrm{C}-\mathrm{O}$ bond formation $(\mathrm{OB}-\mathrm{C} 2$, see Figure 3 ).

\begin{tabular}{cc}
\hline Species & Bond-Order \\
\hline TS1 & 0.05 \\
TS3 & 0.18 \\
TS5 & 0.04 \\
TS5a & 0.04 \\
TS5b & 0.04 \\
TS7 & 0.18 \\
TS7a & 0.17 \\
TS7b & 0.19 \\
$\mathbf{1 0}$ & 0.96 \\
$\mathbf{1 0 a}$ & 0.96 \\
$\mathbf{1 0 b}$ & 0.95 \\
\hline
\end{tabular}


Supplemental Table S2: Energies and bond lengths of transition states with the $\omega$ B97X-D functional and either Ahlrich's def2-TZVP or Pople's 6-31G(d) basis sets.

\begin{tabular}{|c|c|c|c|}
\hline \multicolumn{4}{|c|}{ Energies of calculated transition state structures $\left(\Delta \mathrm{G}^{\ddagger}\right)$} \\
\hline & $\begin{array}{l}\text { DLPNO-CCSD(T)/ cc-pVQZ// } \\
\text { wB97X-D/def2-TZVP }\end{array}$ & $\omega B 97 X-D / d e f 2-T Z V P$ & $\omega \mathrm{B} 97 \mathrm{X}-\mathrm{D} / 6-31 \mathrm{G}(\mathrm{d})$ \\
\hline TS-1 & 28.2 & 30.5 & 28.6 \\
\hline TS-2 & 31.5 & 31.7 & 30.0 \\
\hline TS-5 & 33.0 & 34.2 & 32.5 \\
\hline \multicolumn{4}{|c|}{ Bond lengths of calculated transition state structures $(\AA)$} \\
\hline & $\omega \mathrm{B} 97 \mathrm{X}-\mathrm{D} / \mathrm{def} 2-\mathrm{TZVP}$ & $\omega \mathrm{B} 9$ & $\mathrm{X}-\mathrm{D} / 6-31 \mathrm{G}(\mathrm{d})$ \\
\hline \multicolumn{4}{|l|}{ TS-1 } \\
\hline$C 2-C 1$ & 1.96 & & 1.98 \\
\hline$C 7-C 4$ & 2.63 & & 2.62 \\
\hline $\mathrm{O} 8-\mathrm{C} 2$ & 2.85 & & 2.86 \\
\hline \multicolumn{4}{|l|}{ TS-2 } \\
\hline$C 2-C 1$ & 1.95 & & 1.96 \\
\hline$C 5-C 2$ & 2.92 & & 2.93 \\
\hline C7-C4 & 3.26 & & 3.25 \\
\hline \multicolumn{4}{|l|}{ TS-5 } \\
\hline$C 2-C 1$ & 1.92 & & 1.93 \\
\hline$C 5-C 6$ & 3.11 & & 3.11 \\
\hline$C 7-C 4$ & 3.02 & & 3.03 \\
\hline$O 8-C 2$ & 2.84 & & 2.85 \\
\hline
\end{tabular}


Supplemental Figures

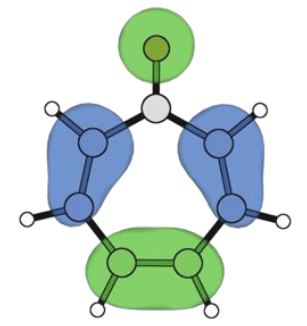

HOMO

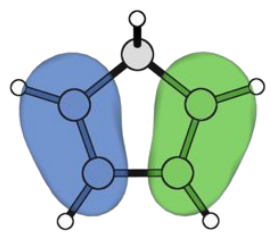

HOMO

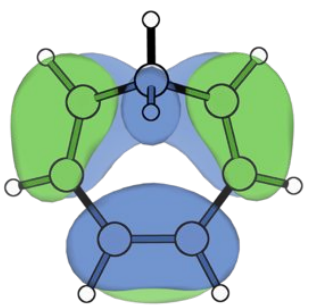

HOMO<smiles>O=c1cccccc1</smiles>

1

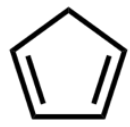

2

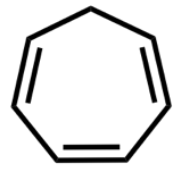

7

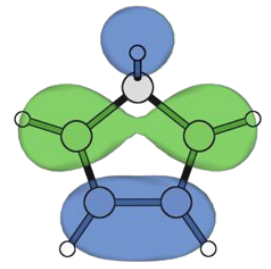

LUMO

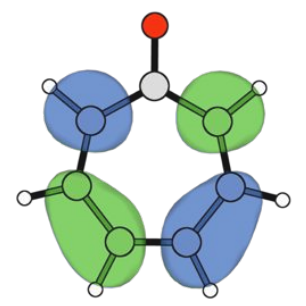

LUMO

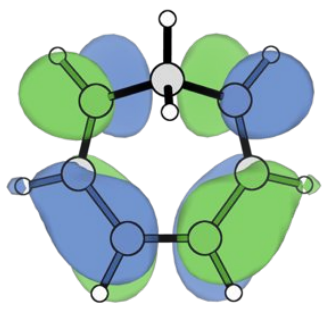

LUMO

Supplemental Figure S1. Highest-occupied and lowest-unoccupied molecular orbital (HOMO and LUMO) calculations for tropone (1), cyclopentadiene (2), and cycloheptatriene (7). 


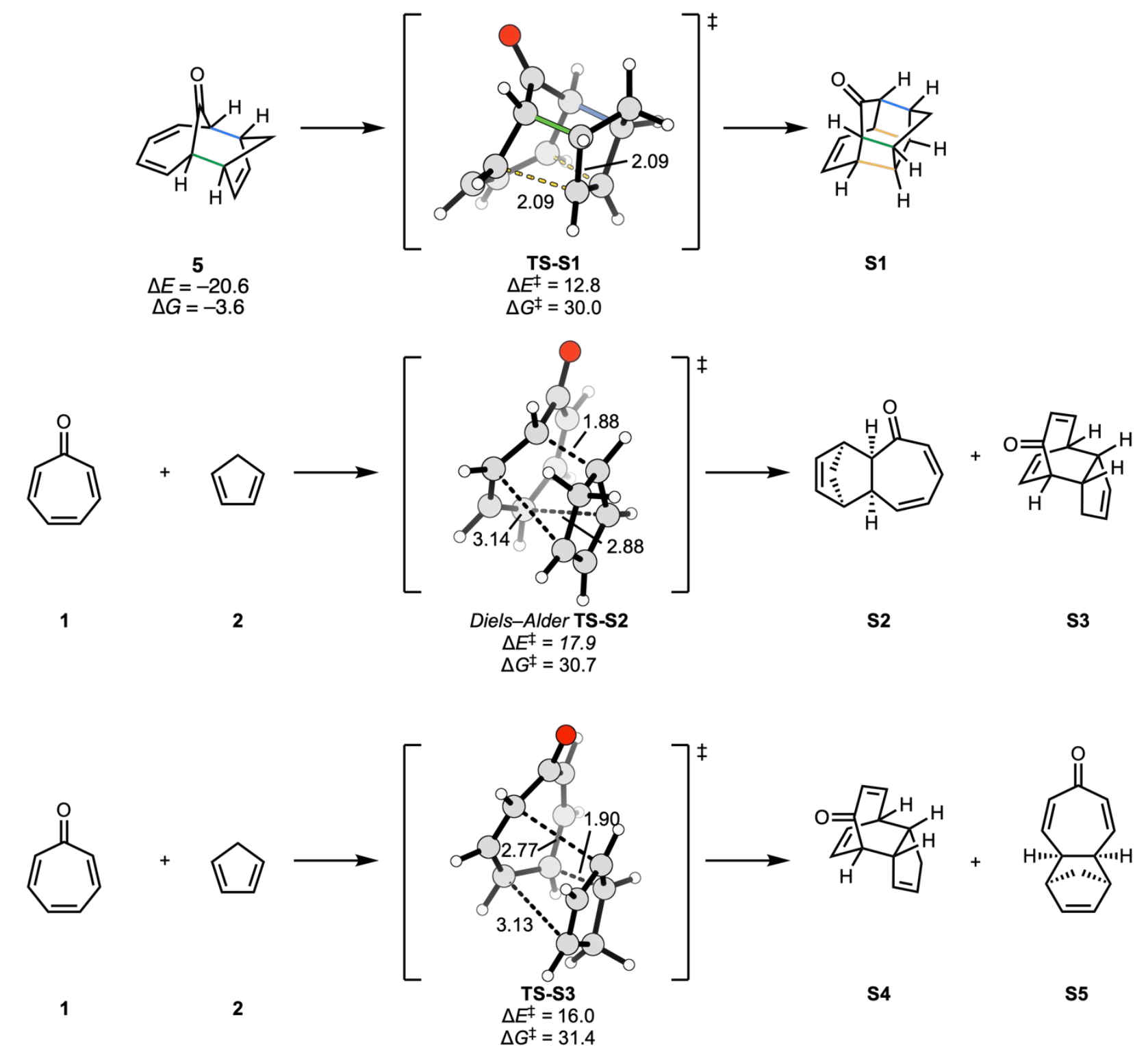

Supplemental Figure S2. Calculated reactions of cyclopentadiene (2) and tropone (1) at the

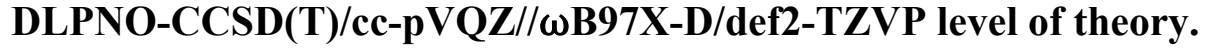




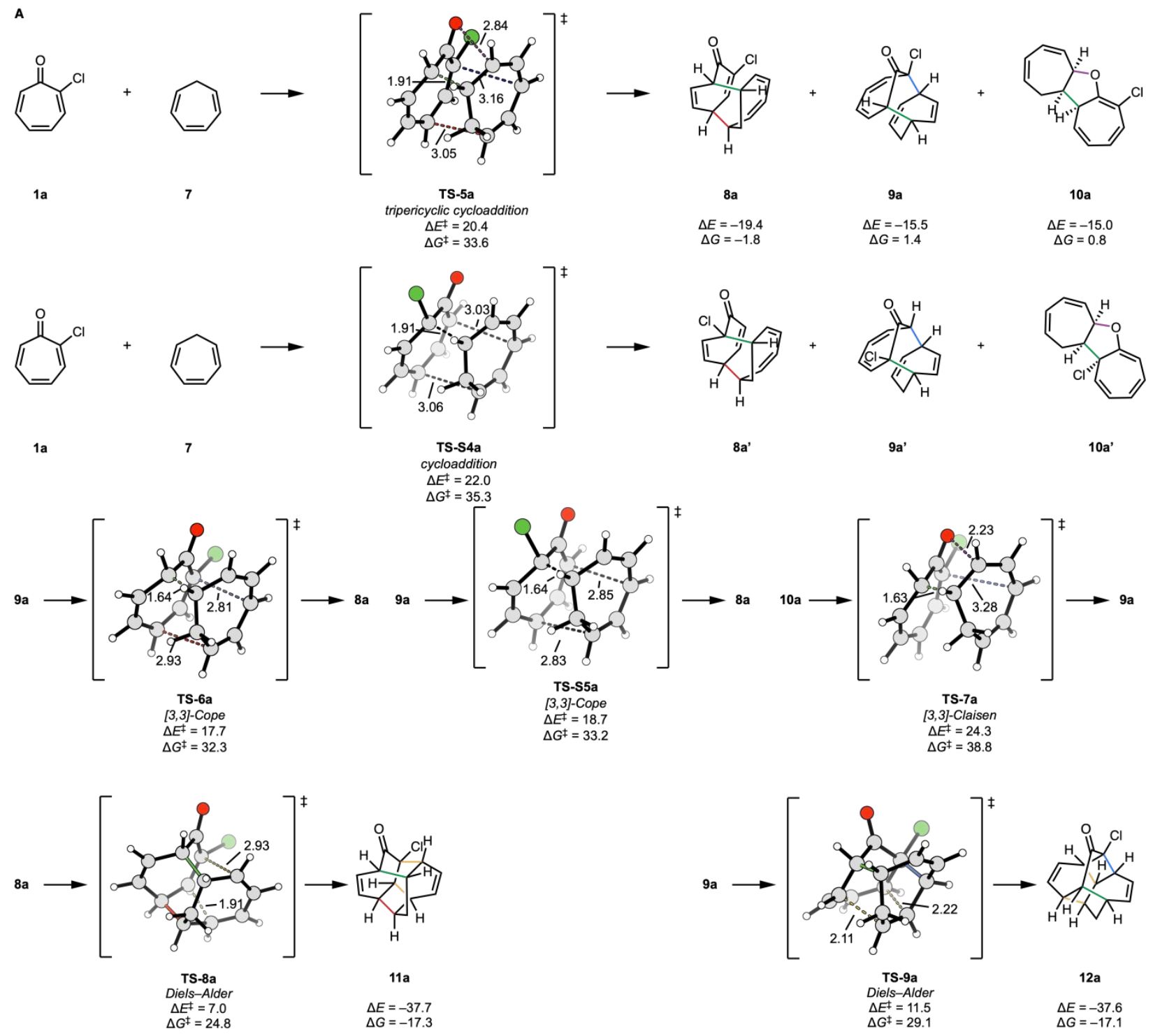

Supplemental Figure S3. Calculated reactions of cycloheptatriene (2) and substituted tropones (1a, 1b) at the DLPNO-CCSD(T)/cc-pVQZ//wB97X-D/def2-TZVP level of theory. Reaction surface of (A) 2-chloro tropone, (B) and 2-methoxy tropone. 


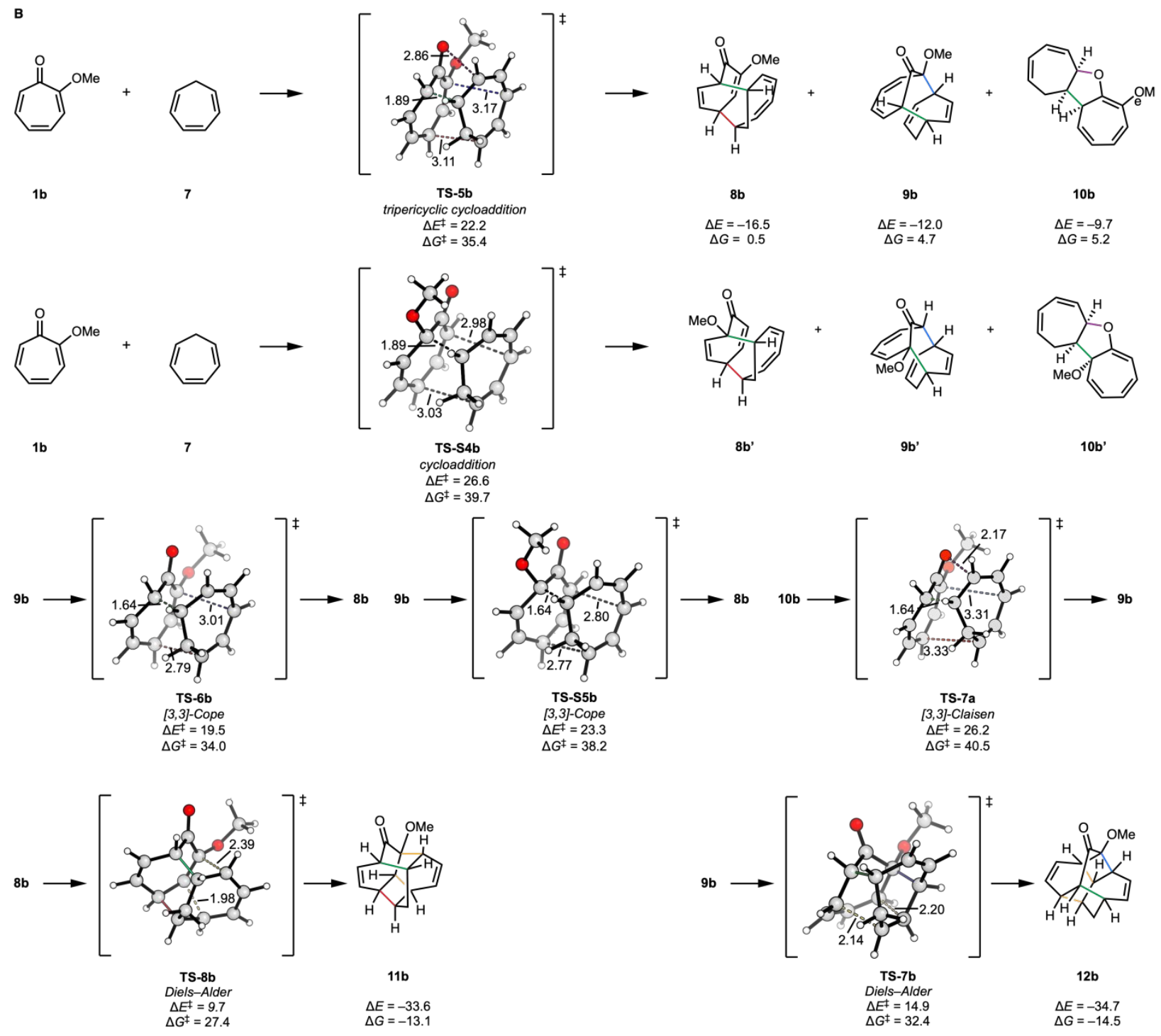

Supplemental Figure S3. Calculated reactions of cycloheptatriene (2) and substituted tropones $(1 \mathrm{a}, 1 \mathrm{~b})$ at the DLPNO-CCSD(T)/cc-pVQZ// $\omega \mathrm{B} 97 \mathrm{X}-\mathrm{D} / \mathrm{def2}-\mathrm{TZVP}$ level of theory. Reaction surface of (A) 2-chloro tropone, (B) and 2-methoxy tropone. 
A

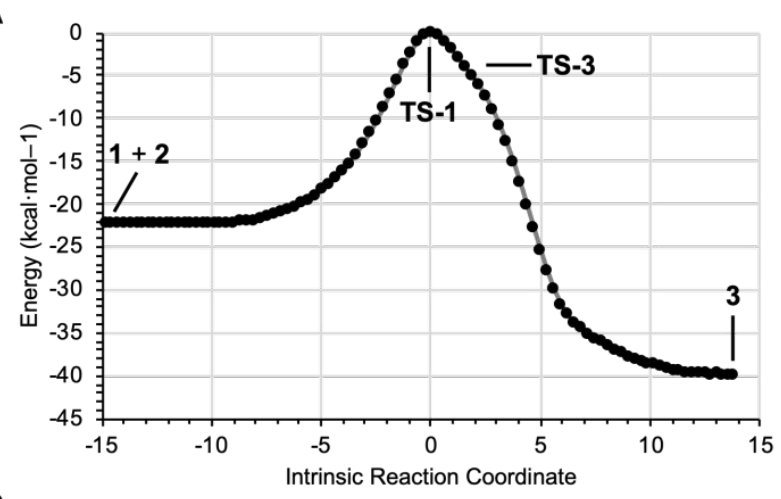

C

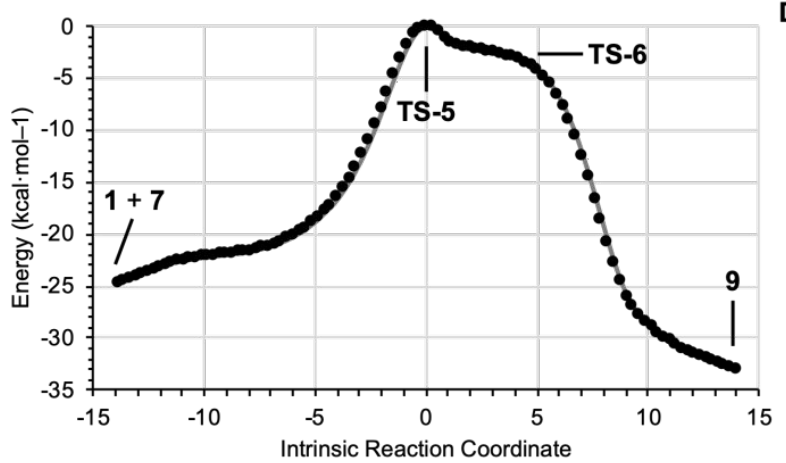

E

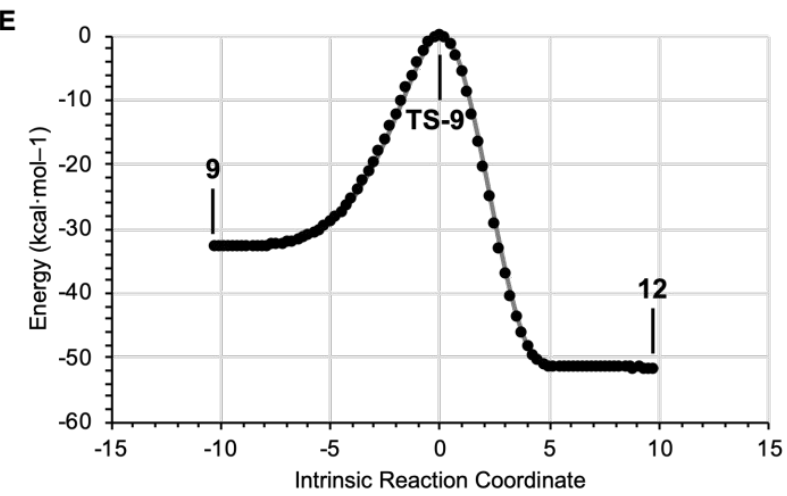

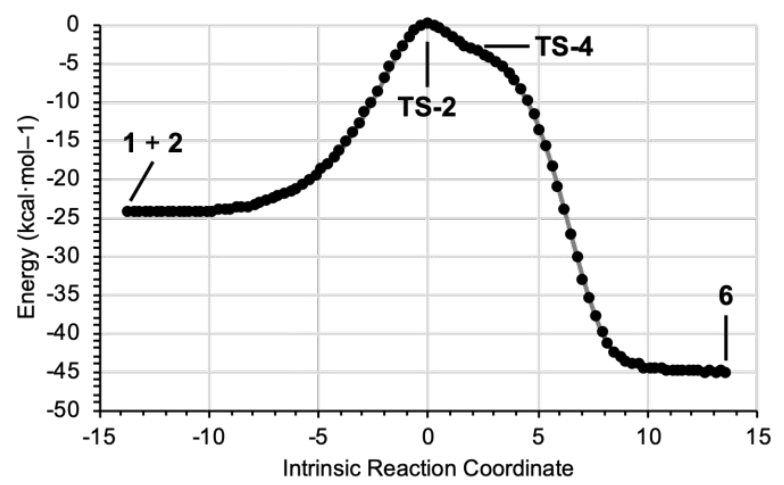

D

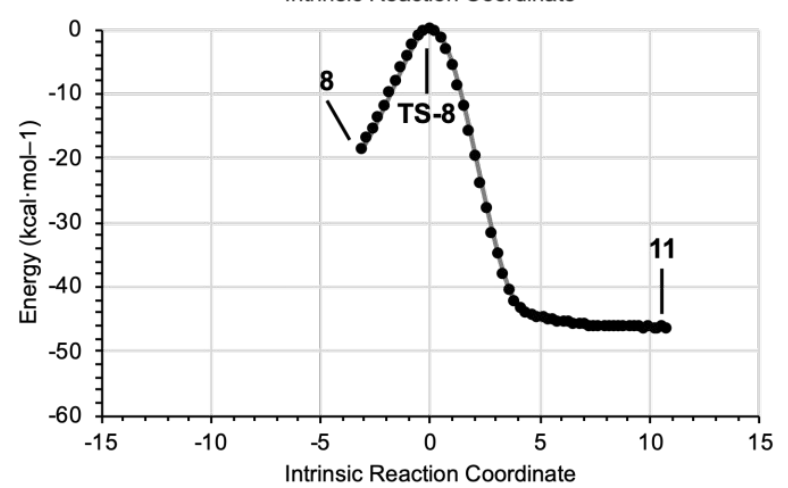

Supplemental Figure S4. Intrinsic reaction coordinate calculations.

(A) TS-1 connects 1 and 2 to 3 by way of TS-3, (B) TS-2 connects 1 and 2 to 6 by way of TS-4,

(C) TS-5 connects 1 and 7 to 9 by way of TS-6, (D) TS-8 connects 8 to 11, (E) TS-9 connects 9 to 12 . 

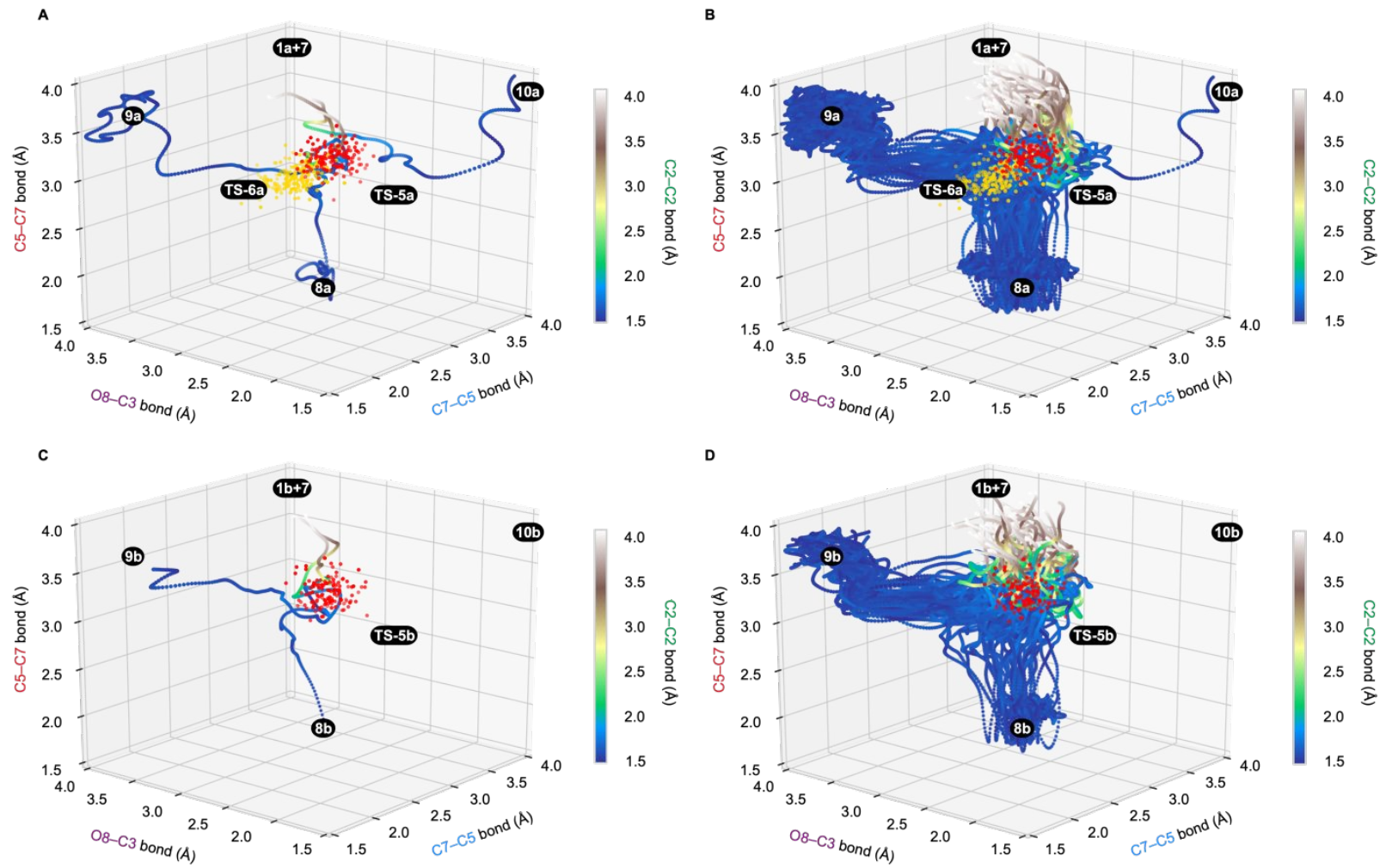

\section{Supplemental Figure S5. Quasi-classical reaction dynamics simulations.}

Trajectories shown as bond length plots of cycloheptatriene (7) reacting with 2-chloro tropone (1a) $(\mathbf{A}, \mathbf{B})$, or 2-methoxy tropone (1b) $(\mathbf{C}, \mathbf{D})$. In panels A and C, single randomly-selected trajectories are draw that lead to each product. In panels B and D, all trajectories are plotted; 177 and 106 trajectories for $\mathbf{7}$ and $\mathbf{1 a}$ and $\mathbf{7}$ and $\mathbf{1 b}$, respectively. 


\section{Cartesian coordinates of calculated structures}

Coordinates of calculated structures were supplied to the publisher as xyz files, and pasted below. Energies are reported in this section uncorrected directly from the output file at the optimization level of theory.

\section{Cyclopentadiene (2) and tropone (1) calculated structures}

\section{1}

$\begin{array}{lr}\text { E } & -271.511346 \\ \text { H } & -271.375615 \\ \text { G } & -271.411304 \\ \text { Imag. Freq. } & 1.000\end{array}$

Cartesian coordinates

$\begin{array}{lrcc}\mathrm{C} & 0.000000 & 0.674517 & -1.838414 \\ \mathrm{C} & -0.000000 & -0.674517 & -1.838414 \\ \mathrm{C} & -0.000000 & -1.557823 & -0.711027 \\ \mathrm{C} & 0.000000 & 1.557823 & -0.711027 \\ \mathrm{C} & -0.000000 & -1.283334 & 0.607818 \\ \mathrm{C} & 0.000000 & 1.283334 & 0.607818 \\ \mathrm{H} & 0.000000 & 1.162356 & -2.806592 \\ \mathrm{H} & -0.000000 & -1.162356 & -2.806592 \\ \mathrm{H} & -0.000000 & -2.612042 & -0.969086 \\ \mathrm{H} & 0.000000 & 2.612042 & -0.969086 \\ \mathrm{H} & -0.000000 & -2.122087 & 1.295537 \\ \mathrm{H} & 0.000000 & 2.122087 & 1.295537 \\ \mathrm{C} & 0.000000 & -0.000000 & 1.320852 \\ \mathrm{O} & 0.000000 & -0.000000 & 2.5418312\end{array}$

2

$\begin{array}{lr}\text { E } & -194.104262 \\ \mathrm{H} & -194.005943 \\ \mathrm{G} & -194.037578 \\ \text { Imag. Freq. } & 1.000\end{array}$

Cartesian coordinates

C $\quad \begin{array}{llll}\text { C } & 1.172304 & -0.279765 & -0.000041\end{array}$

$\begin{array}{llll}\text { C } & 0.732279 & 0.984639 & -0.000017\end{array}$

C $\quad-0.732033 \quad 0.984822-0.000043$

$\begin{array}{llll}\text { C } & -1.172373 & -0.279472 & -0.000047\end{array}$

$\begin{array}{llll}\text { C } & -0.000151 & -1.210543 & 0.000097\end{array}$ 


$\begin{array}{rrrr}\mathrm{H} & -0.000230 & -1.868033 & -0.876503 \\ \mathrm{H} & -0.000244 & -1.867678 & 0.876980 \\ \mathrm{H} & -2.202133 & -0.605078 & -0.000058 \\ \mathrm{H} & -1.346415 & 1.874329 & -0.000049 \\ \mathrm{H} & 1.346882 & 1.873994 & -0.000015 \\ \mathrm{H} & 2.201984 & -0.605624 & -0.000054\end{array}$

\section{3}

$\begin{array}{lr}\text { E } & -539.680620 \\ \text { H } & -539.458321 \\ \text { G } & -539.502433 \\ \text { Imag. Freq. } & 1.000\end{array}$

Cartesian coordinates

$\begin{array}{lrrr}\mathrm{C} & -0.061733 & -0.000035 & 1.223469 \\ \mathrm{O} & -0.136414 & -0.000069 & 2.423645 \\ \mathrm{C} & -0.020961 & 1.289290 & 0.418437 \\ \mathrm{C} & -1.380943 & 1.596520 & -0.140919 \\ \mathrm{C} & -2.338192 & 0.733208 & -0.492661 \\ \mathrm{C} & -2.338199 & -0.733175 & -0.492683 \\ \mathrm{C} & -1.380952 & -1.596509 & -0.140989 \\ \mathrm{C} & -0.020958 & -1.289315 & 0.418362 \\ \mathrm{H} & 0.252907 & -2.079669 & 1.118768 \\ \mathrm{C} & 1.085989 & -1.175260 & -0.684060 \\ \mathrm{C} & 2.352591 & -0.664977 & -0.027106 \\ \mathrm{C} & 2.352591 & 0.664975 & -0.027067 \\ \mathrm{C} & 1.085990 & 1.175298 & -0.683992 \\ \mathrm{H} & 1.192854 & 2.129730 & -1.198807 \\ \mathrm{C} & 0.758683 & 0.000045 & -1.608695 \\ \mathrm{H} & 1.441102 & 0.000070 & -2.459720 \\ \mathrm{H} & -0.263157 & 0.000056 & -1.980590 \\ \mathrm{H} & 3.080850 & 1.299552 & 0.459730 \\ \mathrm{H} & 3.080850 & -1.299583 & 0.459653 \\ \mathrm{H} & 1.192853 & -2.129662 & -1.198931 \\ \mathrm{H} & -1.594817 & -2.652181 & -0.269879 \\ \mathrm{H} & -3.256854 & -1.169031 & -0.870539 \\ \mathrm{H} & -3.256834 & 1.169085 & -0.870525 \\ \mathrm{H} & -1.594788 & 2.652199 & -0.269783 \\ \mathrm{H} & 0.252902 & 2.079604 & 1.118888 \\ & & & \\ 4 & & & \\ & & & \\ \mathrm{E} & -539.671697 & \\ \mathrm{H} & -539.449935 & \\ & & & \\ & & & \\ & & & \\ & & & \\ & & & \\ & & & \end{array}$


$\begin{array}{lr}\text { G } & -539.495828 \\ \text { Imag. Freq. } & 1.000\end{array}$

Cartesian coordinates

$\begin{array}{rrrr}\mathrm{C} & 0.469376 & -0.922662 & 0.009430 \\ \mathrm{O} & -0.722242 & -1.515934 & -0.197119 \\ \mathrm{C} & -1.740457 & -0.857670 & 0.581500 \\ \mathrm{C} & -2.992385 & -0.687598 & -0.221771 \\ \mathrm{C} & -3.087538 & 0.550583 & -0.683265 \\ \mathrm{C} & -1.919257 & 1.405511 & -0.282034 \\ \mathrm{H} & -1.280864 & 1.575338 & -1.154091 \\ \mathrm{H} & -2.218187 & 2.388988 & 0.084717 \\ \mathrm{C} & -1.213574 & 0.566523 & 0.806066 \\ \mathrm{H} & -1.515611 & 0.931657 & 1.786599 \\ \mathrm{C} & 0.313838 & 0.398509 & 0.728456 \\ \mathrm{C} & 1.066674 & 1.501746 & 0.037659 \\ \mathrm{C} & 2.375635 & 1.473245 & -0.231222 \\ \mathrm{C} & 3.271705 & 0.349841 & -0.079528 \\ \mathrm{C} & 2.929028 & -0.953252 & -0.156445 \\ \mathrm{C} & 1.619812 & -1.523430 & -0.330796 \\ \mathrm{H} & 1.556079 & -2.533352 & -0.717184 \\ \mathrm{H} & 3.742601 & -1.671558 & -0.142219 \\ \mathrm{H} & 4.328129 & 0.584060 & -0.012464 \\ \mathrm{H} & 2.819691 & 2.379291 & -0.632443 \\ \mathrm{H} & 0.512350 & 2.400172 & -0.206848 \\ \mathrm{H} & 0.715963 & 0.276956 & 1.741722 \\ \mathrm{H} & -3.882228 & 0.914892 & -1.321908 \\ \mathrm{H} & -3.681778 & -1.496206 & -0.419759 \\ \mathrm{H} & -1.875344 & -1.430846 & 1.502535\end{array}$

5

$\begin{array}{lr}\mathrm{E} & -539.672067 \\ \mathrm{H} & -539.449866 \\ \mathrm{G} & -539.494080 \\ \text { Imag. Freq. } & 1.000\end{array}$

Cartesian coordinates
$\begin{array}{llll}\text { C } & -0.330437 & 1.363419 & -0.000012\end{array}$
$\begin{array}{llll}\text { O } & -0.761244 & 2.487218 & -0.000022\end{array}$
$\begin{array}{llll}\text { C } & -0.038016 & 0.615985 & -1.288723\end{array}$
$\begin{array}{llll}\text { C } & -1.181292 & -0.308285 & -1.601348\end{array}$
$\begin{array}{llll}\text { C } & -1.987511 & -0.925279 & -0.733491\end{array}$
$\begin{array}{llll}\text { C } & -1.987515 & -0.925262 & 0.733503\end{array}$ 


$\begin{array}{lrrr}\mathrm{C} & -1.181297 & -0.308254 & 1.601351 \\ \mathrm{C} & -0.038017 & 0.616007 & 1.288713 \\ \mathrm{H} & 0.018742 & 1.377591 & 2.067307 \\ \mathrm{C} & 1.359077 & -0.105793 & 1.175688 \\ \mathrm{C} & 1.217368 & -1.521774 & 0.662674 \\ \mathrm{C} & 1.217368 & -1.521786 & -0.662644 \\ \mathrm{H} & 1.051236 & -2.379560 & -1.298617 \\ \mathrm{C} & 1.359079 & -0.105815 & -1.175684 \\ \mathrm{H} & 1.881050 & -0.036646 & -2.128854 \\ \mathrm{C} & 2.118038 & 0.533222 & -0.000004 \\ \mathrm{H} & 2.092886 & 1.625406 & -0.000014 \\ \mathrm{H} & 3.158095 & 0.207753 & 0.000001 \\ \mathrm{H} & 1.051233 & -2.379536 & 1.298662 \\ \mathrm{H} & 1.881049 & -0.036607 & 2.128856 \\ \mathrm{H} & -1.353886 & -0.491995 & 2.656408 \\ \mathrm{H} & -2.758211 & -1.553967 & 1.166733 \\ \mathrm{H} & -2.758200 & -1.553999 & -1.166711 \\ \mathrm{H} & -1.353873 & -0.492051 & -2.656402 \\ \mathrm{H} & 0.018746 & 1.377555 & -2.067330\end{array}$

\section{6}

E $\quad-539.686327$

$\mathrm{H} \quad-539.464122$

G $\quad-539.509359$

Imag. Freq. $\quad 1.000$

Cartesian coordinates

$\begin{array}{lrrr}\mathrm{C} & 1.338896 & 1.040007 & 0.327008 \\ \mathrm{O} & 1.935339 & 2.092590 & 0.375197 \\ \mathrm{C} & 0.962642 & 0.336254 & 1.571981 \\ \mathrm{C} & 0.348429 & -0.844031 & 1.628256 \\ \mathrm{C} & -0.052705 & -1.680336 & 0.440169 \\ \mathrm{C} & 1.119215 & -1.935204 & -0.482796 \\ \mathrm{C} & 1.585431 & -0.917198 & -1.187982 \\ \mathrm{C} & 0.934431 & 0.433586 & -1.014141 \\ \mathrm{H} & 1.288970 & 1.137510 & -1.765661 \\ \mathrm{C} & -0.605190 & 0.278563 & -1.144902 \\ \mathrm{H} & -0.789285 & 0.140094 & -2.209874 \\ \mathrm{C} & -1.154675 & -0.968422 & -0.400623 \\ \mathrm{C} & -2.312258 & -0.435195 & 0.392578 \\ \mathrm{C} & -2.462676 & 0.873286 & 0.260421 \\ \mathrm{C} & -1.436599 & 1.487002 & -0.646904 \\ \mathrm{H} & -0.832424 & 2.227500 & -0.114733 \\ \mathrm{H} & -1.903431 & 2.021261 & -1.477856\end{array}$




$\begin{array}{rrrc}\mathrm{H} & -3.229201 & 1.459699 & 0.750949 \\ \mathrm{H} & -2.937223 & -1.072965 & 1.005714 \\ \mathrm{H} & -1.499112 & -1.713899 & -1.121986 \\ \mathrm{H} & 2.422934 & -1.018878 & -1.865863 \\ \mathrm{H} & 1.539424 & -2.930404 & -0.552749 \\ \mathrm{H} & -0.454725 & -2.625999 & 0.804010 \\ \mathrm{H} & 0.098267 & -1.239695 & 2.607167 \\ \mathrm{H} & 1.223451 & 0.865186 & 2.480924\end{array}$

\section{TS-1}

$\begin{array}{lr}\mathrm{E} & -539.616995 \\ \mathrm{H} & -539.398915 \\ \mathrm{G} & -539.444926 \\ \text { Imag. Freq. } & -481.091\end{array}$

Cartesian coordinates

$\begin{array}{lrrr}\mathrm{C} & -0.065553 & -0.291605 & 1.273246 \\ \mathrm{C} & 0.222921 & 1.092296 & 0.821740 \\ \mathrm{C} & 0.473158 & -1.435620 & 0.576090 \\ \mathrm{C} & 1.492062 & 1.532720 & 0.349683 \\ \mathrm{C} & 1.646379 & -1.538785 & -0.162971 \\ \mathrm{C} & 2.485702 & 0.817379 & -0.264328 \\ \mathrm{C} & 2.543181 & -0.557545 & -0.535298 \\ \mathrm{H} & 1.669277 & 2.598919 & 0.447948 \\ \mathrm{H} & 3.348403 & 1.391396 & -0.586085 \\ \mathrm{H} & 3.416208 & -0.894071 & -1.082744 \\ \mathrm{H} & 1.896297 & -2.544455 & -0.485898 \\ \mathrm{H} & -0.004857 & -2.360206 & 0.876086 \\ \mathrm{H} & -0.253304 & 1.794376 & 1.496883 \\ \mathrm{O} & -0.913714 & -0.437338 & 2.147154 \\ \mathrm{C} & -0.650867 & 0.288823 & -1.668000 \\ \mathrm{H} & 0.424648 & 0.182848 & -1.793534 \\ \mathrm{H} & -1.054389 & 0.611661 & -2.636385 \\ \mathrm{C} & -1.363373 & -0.949800 & -1.243526 \\ \mathrm{H} & -1.216847 & -1.916251 & -1.700726 \\ \mathrm{C} & -2.392074 & -0.603682 & -0.400982 \\ \mathrm{H} & -3.130528 & -1.280282 & 0.002207 \\ \mathrm{C} & -1.096307 & 1.281849 & -0.616391 \\ \mathrm{H} & -0.984661 & 2.344529 & -0.785024 \\ \mathrm{C} & -2.274172 & 0.747600 & -0.055735 \\ \mathrm{H} & -2.926876 & 1.268457 & 0.628863\end{array}$

TS-2 


$\begin{array}{lr}\text { E } & -539.613698 \\ \mathrm{H} & -539.396041 \\ \mathrm{G} & -539.442955 \\ \text { Imag. Freq. } & -431.035\end{array}$

Cartesian coordinates

$\begin{array}{lrrr}\mathrm{C} & 0.150846 & 1.489047 & 0.391524 \\ \mathrm{C} & 0.442515 & 0.975691 & -0.983728 \\ \mathrm{C} & 0.505788 & 0.706704 & 1.552190 \\ \mathrm{C} & 1.691276 & 0.412824 & -1.352679 \\ \mathrm{C} & 1.232325 & -0.444312 & 1.590560 \\ \mathrm{C} & 2.336433 & -0.581959 & -0.666437 \\ \mathrm{C} & 1.970802 & -1.089917 & 0.578775 \\ \mathrm{H} & 2.066418 & 0.669282 & -2.336922 \\ \mathrm{H} & 3.167690 & -1.070337 & -1.163932 \\ \mathrm{H} & 2.479191 & -1.996245 & 0.889366 \\ \mathrm{H} & 1.272986 & -0.936464 & 2.557878 \\ \mathrm{H} & 0.103774 & 1.097232 & 2.479198 \\ \mathrm{H} & 0.060904 & 1.702272 & -1.695077 \\ \mathrm{O} & -0.449840 & 2.552763 & 0.491446 \\ \mathrm{C} & -2.181626 & 0.183547 & -0.673116 \\ \mathrm{H} & -3.019285 & -0.047911 & -1.341630 \\ \mathrm{H} & -2.185042 & 1.262521 & -0.513641 \\ \mathrm{C} & -2.312457 & -0.611494 & 0.576935 \\ \mathrm{H} & -3.006625 & -0.375416 & 1.370061 \\ \mathrm{C} & -1.471336 & -1.670601 & 0.539944 \\ \mathrm{H} & -1.392781 & -2.440036 & 1.294396 \\ \mathrm{C} & -0.917026 & -0.383995 & -1.299795 \\ \mathrm{H} & -0.801988 & -0.351744 & -2.375100 \\ \mathrm{C} & -0.644394 & -1.583062 & -0.612203 \\ \mathrm{H} & 0.034603 & -2.350087 & -0.947992\end{array}$

\section{TS-3}

$\begin{array}{ll}\text { E } & -539.617266 \\ \mathrm{H} & -539.398183 \\ \mathrm{G} & -539.442560\end{array}$

Imag. Freq. $\quad-224.637$

Cartesian coordinates
$\begin{array}{llll}\text { C } & -0.092217 & -0.271955 & 1.195273\end{array}$
$\begin{array}{llll}\text { C } & 0.180622 & 1.119163 & 0.678552\end{array}$
$\begin{array}{llll}\text { C } & 0.520355 & -1.431413 & 0.722787\end{array}$
$\begin{array}{llll}\text { C } & 1.529397 & 1.538931 & 0.247552\end{array}$ 


$\begin{array}{lrrr}\mathrm{C} & 1.731068 & -1.571263 & -0.005501 \\ \mathrm{C} & 2.533850 & 0.795632 & -0.249188 \\ \mathrm{C} & 2.599094 & -0.623901 & -0.444407 \\ \mathrm{H} & 1.713974 & 2.605662 & 0.331210 \\ \mathrm{H} & 3.429445 & 1.337084 & -0.537663 \\ \mathrm{H} & 3.477884 & -0.979851 & -0.969921 \\ \mathrm{H} & 1.994229 & -2.596958 & -0.243704 \\ \mathrm{H} & 0.046729 & -2.342692 & 1.068661 \\ \mathrm{H} & -0.161767 & 1.796473 & 1.461912 \\ \mathrm{O} & -1.161553 & -0.310765 & 1.854163 \\ \mathrm{C} & -0.640149 & 0.322452 & -1.640667 \\ \mathrm{H} & 0.424341 & 0.126288 & -1.763165 \\ \mathrm{H} & -0.994573 & 0.764934 & -2.578123 \\ \mathrm{C} & -1.441327 & -0.897211 & -1.338613 \\ \mathrm{H} & -1.328062 & -1.830296 & -1.872588 \\ \mathrm{C} & -2.441644 & -0.611742 & -0.454286 \\ \mathrm{H} & -3.221508 & -1.288638 & -0.140419 \\ \mathrm{C} & -0.973366 & 1.237085 & -0.453273 \\ \mathrm{H} & -1.076559 & 2.291701 & -0.700980 \\ \mathrm{C} & -2.197479 & 0.635965 & 0.126657 \\ \mathrm{H} & -2.860939 & 1.151960 & 0.802160\end{array}$

\section{TS-4}

$\begin{array}{lr}\mathrm{E} & -539.618015 \\ \mathrm{H} & -539.399309 \\ \mathrm{G} & -539.443843 \\ \text { Imag. Freq. } & -190.955\end{array}$

Cartesian coordinates

$\begin{array}{lrrr}\mathrm{C} & -0.014137 & 1.448702 & 0.451060 \\ \mathrm{C} & 0.266857 & 0.961929 & -0.966391 \\ \mathrm{C} & 0.147133 & 0.526616 & 1.558749 \\ \mathrm{C} & 1.668671 & 0.583222 & -1.241681 \\ \mathrm{C} & 1.022037 & -0.549868 & 1.601612 \\ \mathrm{C} & 2.338651 & -0.352769 & -0.554235 \\ \mathrm{C} & 1.883660 & -1.019109 & 0.633162 \\ \mathrm{H} & 2.143804 & 1.043266 & -2.099883 \\ \mathrm{H} & 3.315292 & -0.653928 & -0.919136 \\ \mathrm{H} & 2.443964 & -1.908758 & 0.901594 \\ \mathrm{H} & 0.980871 & -1.143622 & 2.510097 \\ \mathrm{H} & -0.308594 & 0.861296 & 2.482398 \\ \mathrm{H} & -0.023139 & 1.798774 & -1.599627 \\ \mathrm{O} & -0.478441 & 2.567008 & 0.606979 \\ \mathrm{C} & -2.096354 & 0.121568 & -0.636774\end{array}$




$\begin{array}{lrrr}\mathrm{H} & -2.935836 & -0.244419 & -1.237483 \\ \mathrm{H} & -2.256978 & 1.181895 & -0.449295 \\ \mathrm{C} & -1.978596 & -0.725534 & 0.582025 \\ \mathrm{H} & -2.608057 & -0.620088 & 1.453326 \\ \mathrm{C} & -1.209881 & -1.837342 & 0.270973 \\ \mathrm{H} & -1.096755 & -2.712812 & 0.893520 \\ \mathrm{C} & -0.774924 & -0.209953 & -1.358900 \\ \mathrm{H} & -0.834065 & -0.186050 & -2.446044 \\ \mathrm{C} & -0.457562 & -1.565449 & -0.843914 \\ \mathrm{H} & 0.233711 & -2.243690 & -1.319415\end{array}$

\section{TS-S1}

$\begin{array}{lr}\mathrm{E} & -539.619053 \\ \mathrm{H} & -539.399162 \\ \mathrm{G} & -539.441503 \\ \text { Imag. Freq. } & -750.350\end{array}$

Cartesian coordinates

$\begin{array}{lrrr}\mathrm{C} & 0.107770 & 1.463646 & -0.000239 \\ \mathrm{O} & -0.002099 & 2.662295 & -0.000442 \\ \mathrm{C} & 0.199638 & 0.662946 & -1.281252 \\ \mathrm{C} & -1.021118 & -0.233197 & -1.478544 \\ \mathrm{C} & -2.159681 & -0.328226 & -0.694674 \\ \mathrm{C} & -2.159731 & -0.327995 & 0.694630 \\ \mathrm{C} & -1.021226 & -0.232701 & 1.478551 \\ \mathrm{C} & 0.199547 & 0.663372 & 1.281046 \\ \mathrm{H} & 0.318305 & 1.375068 & 2.098738 \\ \mathrm{C} & 1.283106 & -0.430651 & 1.175951 \\ \mathrm{C} & 0.377772 & -1.575066 & 0.695856 \\ \mathrm{C} & 0.377819 & -1.575296 & -0.695308 \\ \mathrm{H} & 0.152211 & -2.449139 & -1.287817 \\ \mathrm{C} & 1.283191 & -0.431042 & -1.175718 \\ \mathrm{H} & 1.775020 & -0.616033 & -2.128846 \\ \mathrm{C} & 2.232825 & -0.203977 & 0.000114 \\ \mathrm{H} & 2.696565 & 0.785606 & -0.000035 \\ \mathrm{H} & 3.021203 & -0.958736 & 0.000268 \\ \mathrm{H} & 0.152121 & -2.448711 & 1.288640 \\ \mathrm{H} & 1.774865 & -0.615329 & 2.129176 \\ \mathrm{H} & -1.140486 & -0.524791 & 2.517872 \\ \mathrm{H} & -3.055357 & -0.702763 & 1.178019 \\ \mathrm{H} & -3.055272 & -0.703156 & -1.178003 \\ \mathrm{H} & -1.140303 & -0.525631 & -2.517776 \\ \mathrm{H} & 0.318450 & 1.374370 & -2.099174\end{array}$




\section{TS-S2}

$\begin{array}{lr}\mathrm{E} & -539.614496 \\ \mathrm{H} & -539.396574 \\ \mathrm{G} & -539.443843 \\ \text { Imag. Freq. } & -372.716\end{array}$

Cartesian coordinates

$\begin{array}{lrrr}\mathrm{C} & 1.767751 & -0.888718 & 0.051169 \\ \mathrm{C} & 0.435759 & -0.883931 & 0.749732 \\ \mathrm{C} & 2.255045 & 0.302749 & -0.608635 \\ \mathrm{C} & -0.010346 & 0.157542 & 1.604984 \\ \mathrm{C} & 1.680564 & 1.528324 & -0.614329 \\ \mathrm{C} & 0.022053 & 1.500375 & 1.283757 \\ \mathrm{C} & 0.606663 & 2.054390 & 0.161569 \\ \mathrm{H} & -0.530562 & -0.132465 & 2.509226 \\ \mathrm{H} & -0.542515 & 2.171337 & 1.921883 \\ \mathrm{H} & 0.380912 & 3.097730 & -0.029067 \\ \mathrm{H} & 2.141641 & 2.256709 & -1.275824 \\ \mathrm{H} & 3.144013 & 0.140522 & -1.206116 \\ \mathrm{H} & 0.303639 & -1.876123 & 1.171077 \\ \mathrm{O} & 2.378496 & -1.944971 & 0.000802 \\ \mathrm{C} & -2.067978 & -1.403459 & 0.037543 \\ \mathrm{H} & -2.638076 & -2.072920 & -0.618746 \\ \mathrm{H} & -1.949431 & -1.913843 & 0.991370 \\ \mathrm{C} & -0.773824 & -1.044196 & -0.682689 \\ \mathrm{H} & -0.240234 & -1.818225 & -1.222782 \\ \mathrm{C} & -1.026248 & 0.197454 & -1.310470 \\ \mathrm{H} & -0.440132 & 0.615557 & -2.112462 \\ \mathrm{C} & -2.764020 & -0.089218 & 0.115082 \\ \mathrm{H} & -3.634863 & 0.100554 & 0.725495 \\ \mathrm{C} & -2.203272 & 0.772194 & -0.766788 \\ \mathrm{H} & -2.555234 & 1.769897 & -0.986008\end{array}$

\section{TS-S3}

$\begin{array}{lr}\mathrm{E} & -539.610980 \\ \mathrm{H} & -539.393088 \\ \mathrm{G} & -539.440314 \\ \text { Imag. Freq. } & -425.274\end{array}$

Cartesian coordinates

$\begin{array}{llll}\text { C } & 2.062357 & -0.477885 & -0.138964\end{array}$

$\begin{array}{llll}\text { C } & 1.209551 & -1.002545 & 0.919632\end{array}$ 


$\begin{array}{lrrr}\mathrm{C} & 2.078428 & 0.964898 & -0.436203 \\ \mathrm{C} & 0.325979 & -0.369703 & 1.769784 \\ \mathrm{C} & 1.075143 & 1.822689 & -0.253983 \\ \mathrm{C} & -0.367263 & 0.802231 & 1.533683 \\ \mathrm{C} & -0.247369 & 1.547319 & 0.331194 \\ \mathrm{H} & 0.033849 & -0.929110 & 2.652299 \\ \mathrm{H} & -1.121348 & 1.103709 & 2.248927 \\ \mathrm{H} & -0.869053 & 2.437722 & 0.322367 \\ \mathrm{H} & 1.210505 & 2.840168 & -0.607837 \\ \mathrm{H} & 2.983203 & 1.299514 & -0.930034 \\ \mathrm{H} & 1.473279 & -2.027244 & 1.162308 \\ \mathrm{O} & 2.828177 & -1.220008 & -0.742963 \\ \mathrm{C} & -2.575808 & 0.496978 & -0.497933 \\ \mathrm{H} & -3.264157 & 0.526250 & -1.351533 \\ \mathrm{H} & -2.867420 & 1.300485 & 0.177171 \\ \mathrm{C} & -1.152763 & 0.578818 & -1.029604 \\ \mathrm{H} & -0.932877 & 1.250388 & -1.850773 \\ \mathrm{C} & -0.681663 & -0.753666 & -1.092021 \\ \mathrm{H} & 0.137609 & -1.099335 & -1.701975 \\ \mathrm{C} & -2.604602 & -0.865440 & 0.110365 \\ \mathrm{H} & -3.398875 & -1.225648 & 0.748003 \\ \mathrm{C} & -1.560999 & -1.590759 & -0.347075 \\ \mathrm{H} & -1.376076 & -2.634446 & -0.138471\end{array}$




\section{Cycloheptatriene (7) and tropone (1) calculated structures}

\begin{tabular}{|c|c|c|c|}
\hline \multicolumn{4}{|l|}{7} \\
\hline \multicolumn{4}{|c|}{ E } \\
\hline \multicolumn{4}{|c|}{$\mathrm{H}$} \\
\hline \multicolumn{4}{|c|}{$\mathrm{G}$} \\
\hline \multicolumn{4}{|c|}{ Imag. Freq. } \\
\hline \multicolumn{4}{|c|}{ Cartesian coordinates } \\
\hline $\mathrm{C}$ & 0.958830 & 1.215723 & -0.200065 \\
\hline $\mathrm{C}$ & -0.342792 & 1.519815 & -0.274856 \\
\hline $\mathrm{C}$ & -1.420584 & 0.675730 & 0.188273 \\
\hline $\mathrm{C}$ & -1.420592 & -0.675713 & 0.188274 \\
\hline $\mathrm{C}$ & -0.342811 & -1.519811 & -0.274856 \\
\hline $\mathrm{C}$ & 0.958815 & -1.215734 & -0.200066 \\
\hline $\mathrm{C}$ & 1.446254 & -0.000009 & 0.533081 \\
\hline $\mathrm{H}$ & 1.687616 & 1.868970 & -0.666790 \\
\hline $\mathrm{H}$ & -0.630820 & 2.460283 & -0.733379 \\
\hline $\mathrm{H}$ & -2.351181 & 1.173669 & 0.440403 \\
\hline $\mathrm{H}$ & -2.351195 & -1.173641 & 0.440404 \\
\hline $\mathrm{H}$ & -0.630850 & -2.460276 & -0.733378 \\
\hline $\mathrm{H}$ & 1.687594 & -1.868990 & -0.666790 \\
\hline $\mathrm{H}$ & 2.532086 & -0.000015 & 0.612016 \\
\hline $\mathrm{H}$ & 1.034027 & -0.000006 & 1.548798 \\
\hline
\end{tabular}

8

E $\quad-617.077957$

$\mathrm{H} \quad-616.818287$

G $\quad-616.866245$

Imag. Freq. $\quad 1.000$

Cartesian coordinates

$\begin{array}{lrrr}\mathrm{C} & -1.751450 & 1.294892 & -0.814355 \\ \mathrm{C} & -1.028298 & 2.136679 & -0.089978 \\ \mathrm{H} & -2.587825 & 1.669286 & -1.392583 \\ \mathrm{H} & -1.272439 & 3.192739 & -0.096761 \\ \mathrm{C} & 0.167862 & 1.706485 & 0.722590 \\ \mathrm{C} & 1.365630 & 1.281363 & -0.212878 \\ \mathrm{H} & 1.868329 & 2.215338 & -0.479220 \\ \mathrm{C} & -1.469026 & -0.183961 & -0.883007 \\ \mathrm{C} & -1.710233 & -0.837574 & 0.474908 \\ \mathrm{O} & -2.509741 & -1.741157 & 0.572976\end{array}$




$\begin{array}{lrrc}\mathrm{C} & -0.952103 & -0.397417 & 1.662513 \\ \mathrm{C} & -0.144151 & 0.653239 & 1.756434 \\ \mathrm{H} & -1.081539 & -1.049613 & 2.518270 \\ \mathrm{H} & 0.376648 & 0.784095 & 2.699525 \\ \mathrm{H} & 0.520770 & 2.572636 & 1.284054 \\ \mathrm{C} & -0.042507 & -0.518436 & -1.482671 \\ \mathrm{H} & -0.246680 & -0.760927 & -2.529826 \\ \mathrm{H} & -2.208451 & -0.656823 & -1.526506 \\ \mathrm{C} & 0.930065 & 0.654943 & -1.537707 \\ \mathrm{H} & 0.486053 & 1.434603 & -2.156547 \\ \mathrm{H} & 1.829159 & 0.319915 & -2.059404 \\ \mathrm{C} & 2.372222 & 0.444623 & 0.519348 \\ \mathrm{C} & 2.464452 & -0.887765 & 0.526824 \\ \mathrm{H} & 3.105900 & 0.993050 & 1.102867 \\ \mathrm{H} & 3.273904 & -1.307120 & 1.115699 \\ \mathrm{C} & 0.544458 & -1.765748 & -0.894391 \\ \mathrm{C} & 1.605756 & -1.895251 & -0.092961 \\ \mathrm{H} & 0.032504 & -2.681865 & -1.173200 \\ \mathrm{H} & 1.865531 & -2.912495 & 0.181811\end{array}$

\section{9}

$\begin{array}{lr}\text { E } & -617.072894 \\ \mathrm{H} & -616.813611 \\ \mathrm{G} & -616.861993 \\ \text { Imag. Freq. } & 1.000\end{array}$

Cartesian coordinates

$\begin{array}{lrrc}\mathrm{C} & 2.000056 & -1.355630 & -0.614757 \\ \mathrm{C} & 2.413414 & -0.709779 & 0.631471 \\ \mathrm{C} & 1.875594 & 0.321892 & 1.283811 \\ \mathrm{C} & 1.034063 & -1.042799 & -1.480700 \\ \mathrm{H} & 2.574391 & -2.244895 & -0.852775 \\ \mathrm{H} & 3.277086 & -1.171695 & 1.097817 \\ \mathrm{H} & 2.336766 & 0.612166 & 2.221911 \\ \mathrm{H} & 0.906985 & -1.710162 & -2.327590 \\ \mathrm{C} & 0.612871 & 1.272929 & -0.629902 \\ \mathrm{O} & 1.004915 & 2.281612 & -1.160783 \\ \mathrm{C} & 0.684759 & 1.141470 & 0.880178 \\ \mathrm{H} & 0.847179 & 2.162788 & 1.226936 \\ \mathrm{C} & 0.093921 & 0.126793 & -1.479545 \\ \mathrm{H} & 0.116764 & 0.543926 & -2.487752 \\ \mathrm{C} & -0.661458 & 0.646269 & 1.525864 \\ \mathrm{C} & -1.628780 & -1.614498 & -0.527341 \\ \mathrm{H} & -2.703505 & -1.830296 & -0.540719\end{array}$




$\begin{array}{llll}\mathrm{H} & -1.168624 & -2.394930 & -1.140422 \\ \mathrm{C} & -1.413990 & -0.258186 & -1.206642 \\ \mathrm{H} & -1.867027 & -0.353127 & -2.194400 \\ \mathrm{C} & -0.718560 & -0.849848 & 1.722913 \\ \mathrm{C} & -1.128952 & -1.779503 & 0.873887 \\ \mathrm{H} & -0.354384 & -1.187992 & 2.686995 \\ \mathrm{H} & -1.098193 & -2.809065 & 1.217908 \\ \mathrm{C} & -2.147075 & 0.834649 & -0.470770 \\ \mathrm{H} & -2.975955 & 1.316900 & -0.976418 \\ \mathrm{C} & -1.826516 & 1.219130 & 0.758039 \\ \mathrm{H} & -2.392105 & 2.014674 & 1.229646 \\ \mathrm{H} & -0.674784 & 1.081477 & 2.526086\end{array}$

10

$\begin{array}{lr}\text { E } & -617.073628 \\ \mathrm{H} & -616.815608 \\ \mathrm{G} & -616.865222 \\ \text { Imag. Freq. } & 1.000\end{array}$

Cartesian coordinates

$\begin{array}{lrrr}\mathrm{C} & 1.213227 & 0.945286 & -0.332714 \\ \mathrm{C} & 2.496505 & 1.335852 & -0.251631 \\ \mathrm{C} & 3.583249 & 0.399255 & -0.213865 \\ \mathrm{C} & 3.552521 & -0.864869 & 0.279154 \\ \mathrm{C} & 2.417394 & -1.484500 & 0.918081 \\ \mathrm{C} & 1.130795 & -1.253112 & 0.618106 \\ \mathrm{C} & 0.767159 & -0.459797 & -0.604749 \\ \mathrm{H} & 1.331744 & -0.859969 & -1.451894 \\ \mathrm{C} & -0.713858 & -0.260911 & -0.970017 \\ \mathrm{H} & -0.752334 & -0.083866 & -2.047849 \\ \mathrm{C} & -1.681971 & -1.389346 & -0.626880 \\ \mathrm{C} & -3.133026 & -0.997335 & -0.705779 \\ \mathrm{C} & -3.702920 & -0.172955 & 0.175362 \\ \mathrm{C} & -2.947112 & 0.497870 & 1.234274 \\ \mathrm{C} & -1.729505 & 1.008310 & 1.049376 \\ \mathrm{C} & -1.066316 & 1.081451 & -0.298906 \\ \mathrm{H} & -1.713029 & 1.664237 & -0.959677 \\ \mathrm{O} & 0.171790 & 1.791878 & -0.205641 \\ \mathrm{H} & -1.202431 & 1.491262 & 1.863995 \\ \mathrm{H} & -3.418112 & 0.594596 & 2.206827 \\ \mathrm{H} & -4.773603 & -0.002031 & 0.134131 \\ \mathrm{H} & -3.746380 & -1.457095 & -1.472377 \\ \mathrm{H} & -1.492408 & -1.716341 & 0.397816 \\ \mathrm{H} & -1.481864 & -2.242663 & -1.275955\end{array}$




$\begin{array}{rrrr}\mathrm{H} & 0.352198 & -1.653710 & 1.254683 \\ \mathrm{H} & 2.638463 & -2.160683 & 1.738094 \\ \mathrm{H} & 4.494883 & -1.398090 & 0.337313 \\ \mathrm{H} & 4.553715 & 0.783963 & -0.511360 \\ \mathrm{H} & 2.717990 & 2.394182 & -0.187485\end{array}$

11

$\begin{array}{lr}\text { E } & -617.105326 \\ \mathrm{H} & -616.844331 \\ \mathrm{G} & -616.888677 \\ \text { Imag. Freq. } & 1.000\end{array}$

Cartesian coordinates

$\begin{array}{lrrr}\mathrm{C} & 1.514327 & -1.117598 & -0.375484 \\ \mathrm{O} & 2.452672 & -1.649657 & -0.898375 \\ \mathrm{C} & 0.073854 & -1.143065 & -0.825848 \\ \mathrm{H} & -0.096686 & -1.938064 & -1.549526 \\ \mathrm{C} & -0.397939 & 0.202529 & -1.417697 \\ \mathrm{H} & -0.335342 & 0.136447 & -2.502385 \\ \mathrm{C} & 0.143167 & 1.561896 & -0.856640 \\ \mathrm{C} & 1.501076 & 1.817523 & -0.278562 \\ \mathrm{C} & 2.103985 & 1.014663 & 0.587453 \\ \mathrm{C} & 1.539160 & -0.336457 & 0.943466 \\ \mathrm{H} & 2.205957 & -0.847302 & 1.636596 \\ \mathrm{C} & 0.064034 & -0.381905 & 1.460364 \\ \mathrm{H} & 0.066980 & -0.751760 & 2.486166 \\ \mathrm{C} & -0.698879 & 0.953739 & 1.481437 \\ \mathrm{C} & -1.047872 & 1.574569 & 0.130344 \\ \mathrm{H} & -1.519985 & 2.544236 & 0.296108 \\ \mathrm{C} & -1.799242 & 0.627653 & -0.853388 \\ \mathrm{C} & -2.664041 & -0.430485 & -0.267509 \\ \mathrm{C} & -2.092302 & -1.403739 & 0.429682 \\ \mathrm{C} & -0.600525 & -1.438853 & 0.530683 \\ \mathrm{H} & -0.277559 & -2.430169 & 0.859758 \\ \mathrm{H} & -2.675648 & -2.189238 & 0.894432 \\ \mathrm{H} & -3.739236 & -0.378828 & -0.391211 \\ \mathrm{H} & -2.333506 & 1.224022 & -1.592667 \\ \mathrm{H} & -0.111872 & 1.678859 & 2.049545 \\ \mathrm{H} & -1.625467 & 0.799948 & 2.038992 \\ \mathrm{H} & 3.067306 & 1.286316 & 1.000490 \\ \mathrm{H} & 1.969757 & 2.762509 & -0.530260 \\ \mathrm{H} & -0.048888 & 2.297469 & -1.638852\end{array}$




$\begin{array}{lr}\text { E } & -617.102991 \\ \mathrm{H} & -616.842091 \\ \mathrm{G} & -616.886771 \\ \text { Imag. Freq. } & 1.000\end{array}$

Cartesian coordinates

$\begin{array}{lrrr}\mathrm{C} & -2.258658 & 0.776713 & -0.667468 \\ \mathrm{C} & -2.563209 & -0.055512 & 0.319691 \\ \mathrm{C} & -1.463739 & -0.552785 & 1.189305 \\ \mathrm{C} & -0.844313 & 1.244733 & -0.802455 \\ \mathrm{H} & -3.011498 & 1.145902 & -1.352822 \\ \mathrm{H} & -3.575705 & -0.405953 & 0.478925 \\ \mathrm{H} & -1.841426 & -1.036969 & 2.088976 \\ \mathrm{H} & -0.811475 & 2.132711 & -1.437462 \\ \mathrm{C} & -0.137688 & -1.198101 & -0.932856 \\ \mathrm{O} & -0.172621 & -2.085940 & -1.748240 \\ \mathrm{C} & -0.406251 & -1.492349 & 0.530109 \\ \mathrm{C} & 0.182527 & 0.230815 & -1.371237 \\ \mathrm{C} & 0.662300 & -0.814330 & 1.426977 \\ \mathrm{C} & 1.215751 & 1.938162 & 0.107004 \\ \mathrm{H} & 1.930842 & 2.004779 & 0.925537 \\ \mathrm{H} & 1.208591 & 2.900277 & -0.409685 \\ \mathrm{C} & 1.574189 & 0.791063 & -0.875590 \\ \mathrm{H} & 2.100852 & 1.187875 & -1.741701 \\ \mathrm{C} & -0.291063 & 0.427107 & 1.544941 \\ \mathrm{C} & -0.200275 & 1.599089 & 0.550071 \\ \mathrm{H} & -0.323948 & 0.832116 & 2.555578 \\ \mathrm{H} & -0.712280 & 2.455140 & 0.992768 \\ \mathrm{C} & 2.460135 & -0.205462 & -0.180782 \\ \mathrm{H} & 3.467373 & -0.344642 & -0.555736 \\ \mathrm{C} & 2.064889 & -0.852721 & 0.906273 \\ \mathrm{H} & 2.749178 & -1.533796 & 1.399941 \\ \mathrm{H} & 0.630950 & -1.303024 & 2.401269 \\ \mathrm{H} & -0.554804 & -2.565092 & 0.634998 \\ \mathrm{H} & 0.156748 & 0.199667 & -2.458561\end{array}$

\section{TS-5}

$\begin{array}{lr}\mathrm{E} & -617.017597 \\ \mathrm{H} & -616.762363 \\ \mathrm{G} & -616.812605 \\ \text { Imag. Freq. } & -446.425\end{array}$


Cartesian coordinates

$\begin{array}{lrrr}\mathrm{C} & -0.068089 & 1.627317 & -0.312450 \\ \mathrm{C} & -0.463544 & 1.156608 & 1.050090 \\ \mathrm{C} & -0.555934 & 1.029784 & -1.532871 \\ \mathrm{C} & -1.726722 & 0.582268 & 1.369532 \\ \mathrm{C} & -1.537786 & 0.091625 & -1.703012 \\ \mathrm{C} & -2.523484 & -0.215123 & 0.585057 \\ \mathrm{C} & -2.372465 & -0.522291 & -0.765498 \\ \mathrm{H} & -2.044526 & 0.708337 & 2.399643 \\ \mathrm{H} & -3.376336 & -0.669562 & 1.079096 \\ \mathrm{H} & -3.081087 & -1.236624 & -1.169117 \\ \mathrm{H} & -1.692714 & -0.231211 & -2.727973 \\ \mathrm{H} & -0.084558 & 1.443627 & -2.415957 \\ \mathrm{H} & -0.159630 & 1.927590 & 1.749796 \\ \mathrm{O} & 0.779758 & 2.510749 & -0.357705 \\ \mathrm{C} & 0.315304 & -1.520175 & 1.458236 \\ \mathrm{C} & 0.870630 & -0.112776 & 1.590420 \\ \mathrm{C} & 0.153571 & -2.124378 & 0.103050 \\ \mathrm{C} & 2.039465 & 0.317734 & 0.934341 \\ \mathrm{C} & 0.800833 & -1.821498 & -1.032088 \\ \mathrm{C} & 2.428318 & 0.012985 & -0.366150 \\ \mathrm{C} & 1.809079 & -0.834634 & -1.256021 \\ \mathrm{H} & 2.604532 & 1.101488 & 1.424360 \\ \mathrm{H} & 3.259697 & 0.587888 & -0.755963 \\ \mathrm{H} & 2.215714 & -0.829193 & -2.261585 \\ \mathrm{H} & 0.528308 & -2.397648 & -1.909698 \\ \mathrm{H} & -0.542775 & -2.955321 & 0.064049 \\ \mathrm{H} & 0.859352 & 0.172913 & 2.638665 \\ \mathrm{H} & 0.930489 & -2.191648 & 2.070233 \\ \mathrm{H} & -0.669582 & -1.521313 & 1.940277\end{array}$

TS-6

$\begin{array}{lr}\mathrm{E} & -617.021491 \\ \mathrm{H} & -616.765749 \\ \mathrm{G} & -616.814112 \\ \text { Imag. Freq. } & -112.224\end{array}$

Cartesian coordinates

$\begin{array}{lrrr}\text { C } & -0.081351 & -1.561233 & -0.544289 \\ \text { C } & 0.301366 & -1.251125 & 0.898125 \\ \text { C } & 0.394363 & -0.804949 & -1.682898 \\ \text { C } & 1.703320 & -0.914615 & 1.215223 \\ \text { C } & 1.406283 & 0.122149 & -1.720099\end{array}$ 


$\begin{array}{cccc}\mathrm{C} & 2.498430 & -0.055966 & 0.548509 \\ \mathrm{C} & 2.236704 & 0.573719 & -0.702360 \\ \mathrm{H} & 2.080985 & -1.321130 & 2.147596 \\ \mathrm{H} & 3.446935 & 0.199438 & 1.010694 \\ \mathrm{H} & 2.943159 & 1.345444 & -0.987004 \\ \mathrm{H} & 1.540835 & 0.621018 & -2.675249 \\ \mathrm{H} & -0.081669 & -1.092784 & -2.611883 \\ \mathrm{H} & 0.027977 & -2.167136 & 1.418572 \\ \mathrm{O} & -0.906090 & -2.448488 & -0.698936 \\ \mathrm{C} & -0.120697 & 1.285930 & 1.615989 \\ \mathrm{C} & -0.695641 & -0.135590 & 1.563236 \\ \mathrm{C} & 0.102718 & 2.041009 & 0.350406 \\ \mathrm{C} & -2.033875 & -0.281207 & 0.974558 \\ \mathrm{C} & -0.639778 & 1.961403 & -0.783734 \\ \mathrm{C} & -2.428001 & 0.230398 & -0.214869 \\ \mathrm{C} & -1.693002 & 1.084295 & -1.073309 \\ \mathrm{H} & -2.705047 & -0.979603 & 1.460280 \\ \mathrm{H} & -3.379788 & -0.112182 & -0.604325 \\ \mathrm{H} & -2.126350 & 1.209025 & -2.059861 \\ \mathrm{H} & -0.345852 & 2.614480 & -1.597987 \\ \mathrm{H} & 0.828871 & 2.843202 & 0.413268 \\ \mathrm{H} & -0.742286 & -0.457940 & 2.604220 \\ \mathrm{H} & -0.775152 & 1.892263 & 2.254554 \\ \mathrm{H} & 0.831070 & 1.228500 & 2.151693\end{array}$

\section{TS-7}

E $\quad-617.015287$

$\mathrm{H} \quad-616.759437$

G $\quad-616.808241$

Imag. Freq. $\quad-241.250$

Cartesian coordinates

$\begin{array}{lrrr}\mathrm{C} & -0.047482 & -1.510434 & 0.091366 \\ \mathrm{C} & -0.366278 & -0.852531 & -1.230116 \\ \mathrm{C} & -0.659631 & -1.283006 & 1.321473 \\ \mathrm{C} & -1.680248 & -0.243938 & -1.516233 \\ \mathrm{C} & -1.769871 & -0.468124 & 1.608360 \\ \mathrm{C} & -2.573922 & 0.276427 & -0.657442 \\ \mathrm{C} & -2.559569 & 0.266915 & 0.773895 \\ \mathrm{H} & -1.925652 & -0.177619 & -2.572239 \\ \mathrm{H} & -3.448398 & 0.743439 & -1.100656 \\ \mathrm{H} & -3.351170 & 0.837984 & 1.246068 \\ \mathrm{H} & -2.010017 & -0.390593 & 2.664287 \\ \mathrm{H} & -0.204077 & -1.821742 & 2.143562 \\ \mathrm{H} & -0.189389 & -1.643084 & -1.960640\end{array}$




$\begin{array}{lrrc}\mathrm{O} & 1.017726 & -2.172527 & 0.000493 \\ \mathrm{C} & 0.562821 & 1.625043 & -1.265973 \\ \mathrm{C} & 0.894468 & 0.146541 & -1.491008 \\ \mathrm{C} & 0.129227 & 2.055200 & 0.097003 \\ \mathrm{C} & 2.054970 & -0.393336 & -0.753676 \\ \mathrm{C} & 0.652984 & 1.642975 & 1.254820 \\ \mathrm{C} & 2.444944 & -0.032116 & 0.538871 \\ \mathrm{C} & 1.739443 & 0.722089 & 1.442635 \\ \mathrm{H} & 2.731399 & -1.031192 & -1.304754 \\ \mathrm{H} & 3.313433 & -0.552039 & 0.924303 \\ \mathrm{H} & 2.121438 & 0.691977 & 2.458267 \\ \mathrm{H} & 0.239424 & 2.057644 & 2.166698 \\ \mathrm{H} & -0.657867 & 2.799098 & 0.137208 \\ \mathrm{H} & 1.102336 & 0.047285 & -2.557228 \\ \mathrm{H} & 1.434005 & 2.225119 & -1.558453 \\ \mathrm{H} & -0.228415 & 1.883708 & -1.974195\end{array}$

\section{TS-8}

$\begin{array}{lr}\mathrm{E} & -617.020573 \\ \mathrm{H} & -616.763221 \\ \mathrm{G} & -616.808922 \\ \text { Imag. Freq. } & -681.014\end{array}$

Cartesian coordinates

$\begin{array}{lrrr}\mathrm{C} & -2.213741 & 0.935256 & -0.674731 \\ \mathrm{C} & -2.598622 & 0.006376 & 0.284733 \\ \mathrm{C} & -1.723864 & -0.780576 & 0.998960 \\ \mathrm{C} & -0.894893 & 1.205399 & -1.014218 \\ \mathrm{H} & -2.952526 & 1.678048 & -0.953906 \\ \mathrm{H} & -3.607208 & 0.096541 & 0.673580 \\ \mathrm{H} & -2.121925 & -1.241858 & 1.898343 \\ \mathrm{H} & -0.766948 & 2.129415 & -1.572971 \\ \mathrm{C} & -0.139269 & -1.209971 & -0.930828 \\ \mathrm{O} & -0.152289 & -2.118152 & -1.721916 \\ \mathrm{C} & -0.442299 & -1.457847 & 0.531865 \\ \mathrm{C} & 0.184125 & 0.202969 & -1.383255 \\ \mathrm{C} & 0.587945 & -0.797134 & 1.471678 \\ \mathrm{C} & 1.298195 & 1.932335 & 0.011625 \\ \mathrm{H} & 2.149837 & 2.128328 & 0.666037 \\ \mathrm{H} & 1.146008 & 2.828847 & -0.591111 \\ \mathrm{C} & 1.584686 & 0.725521 & -0.889621 \\ \mathrm{H} & 2.147606 & 1.050772 & -1.763654 \\ \mathrm{C} & -0.099319 & 0.560454 & 1.652098 \\ \mathrm{C} & 0.045473 & 1.661635 & 0.817869\end{array}$




$\begin{array}{rrrr}\mathrm{H} & -0.583052 & 0.714072 & 2.606175 \\ \mathrm{H} & -0.491077 & 2.548444 & 1.138211 \\ \mathrm{C} & 2.416095 & -0.284637 & -0.143194 \\ \mathrm{H} & 3.420635 & -0.472554 & -0.504614 \\ \mathrm{C} & 1.993843 & -0.900140 & 0.953280 \\ \mathrm{H} & 2.655041 & -1.596648 & 1.456522 \\ \mathrm{H} & 0.529722 & -1.310696 & 2.431930 \\ \mathrm{H} & -0.507364 & -2.541395 & 0.644981 \\ \mathrm{H} & 0.209433 & 0.136059 & -2.471755\end{array}$

\section{TS-9}

$\begin{array}{lr}\mathrm{E} & -617.031363 \\ \mathrm{H} & -616.773887 \\ \mathrm{G} & -616.819304 \\ \text { Imag. Freq. } & -652.121\end{array}$

Cartesian coordinates

$\begin{array}{lrrr}\mathrm{C} & 1.602075 & -1.018555 & -0.403845 \\ \mathrm{O} & 2.513811 & -1.742380 & -0.713058 \\ \mathrm{C} & 0.334291 & -0.942808 & -1.170230 \\ \mathrm{H} & 0.184280 & -1.789241 & -1.830397 \\ \mathrm{C} & -0.270478 & 0.254571 & -1.542757 \\ \mathrm{H} & -0.680442 & 0.285772 & -2.543360 \\ \mathrm{C} & -0.069342 & 1.612979 & -0.864851 \\ \mathrm{C} & 1.275865 & 1.997369 & -0.319591 \\ \mathrm{C} & 1.989988 & 1.227856 & 0.490914 \\ \mathrm{C} & 1.582398 & -0.173922 & 0.860538 \\ \mathrm{H} & 2.322494 & -0.597163 & 1.537170 \\ \mathrm{C} & 0.146753 & -0.355660 & 1.451729 \\ \mathrm{H} & 0.275090 & -0.685902 & 2.486578 \\ \mathrm{C} & -0.715772 & 0.900886 & 1.524189 \\ \mathrm{C} & -1.181462 & 1.474688 & 0.193977 \\ \mathrm{H} & -1.671526 & 2.430323 & 0.402383 \\ \mathrm{C} & -2.136266 & 0.569558 & -0.571793 \\ \mathrm{C} & -2.607981 & -0.681255 & -0.217659 \\ \mathrm{C} & -1.847983 & -1.667662 & 0.396864 \\ \mathrm{C} & -0.509848 & -1.526947 & 0.735599 \\ \mathrm{H} & 0.001997 & -2.464140 & 0.947544 \\ \mathrm{H} & -2.239435 & -2.678085 & 0.356406 \\ \mathrm{H} & -3.535800 & -1.003868 & -0.678525 \\ \mathrm{H} & -2.755579 & 1.116908 & -1.278241 \\ \mathrm{H} & -0.157902 & 1.670190 & 2.061444 \\ \mathrm{H} & -1.599697 & 0.676966 & 2.125250 \\ \mathrm{H} & 2.934302 & 1.589258 & 0.879322\end{array}$


H $\quad 1.638262 \quad 2.992288 \quad-0.552993$

H $\quad-0.379949 \quad 2.369148 \quad-1.586624$ 


\section{Cycloheptatriene (7) and 2-chlorotropone (1a) calculated structures}

\section{1a}

$\begin{array}{lr}\text { E } & -805.148163 \\ \mathrm{H} & -805.038196 \\ \mathrm{G} & -805.079701 \\ \text { Imag. Freq. } & 1.000\end{array}$

Cartesian coordinates

$\begin{array}{lrrc}\mathrm{O} & 1.101508 & 1.972403 & -0.001950 \\ \mathrm{C} & 0.256667 & 1.099437 & 0.000445 \\ \mathrm{C} & -1.157568 & 1.480170 & 0.000990 \\ \mathrm{C} & -2.294230 & 0.754768 & 0.000671 \\ \mathrm{C} & -2.476778 & -0.659352 & -0.000328 \\ \mathrm{C} & -1.516830 & -1.608921 & -0.000715 \\ \mathrm{C} & -0.099879 & -1.449788 & -0.000227 \\ \mathrm{C} & 0.659129 & -0.330977 & 0.000257 \\ \mathrm{H} & 0.457136 & -2.379238 & -0.000335 \\ \mathrm{H} & -1.850549 & -2.640068 & -0.001448 \\ \mathrm{H} & -3.505095 & -1.001051 & -0.000782 \\ \mathrm{H} & -3.213308 & 1.331463 & 0.001233 \\ \mathrm{H} & -1.257949 & 2.559914 & 0.001612 \\ \mathrm{Cl} & 2.372626 & -0.550721 & 0.000515\end{array}$

$8 \mathbf{a}$

E $\quad-1076.686688$

$\mathrm{H} \quad-1076.435189$

G $\quad-1076.486414$

Imag. Freq. $\quad 1.000$

Cartesian coordinates
C $\quad 1.314115$
0.918659
0.610627
$\begin{array}{llll}\mathrm{O} & 2.283884 & 1.131575 & 1.291288\end{array}$
$\begin{array}{llll}\text { C } & 1.384858 & 0.003687 & -0.566069\end{array}$
$\begin{array}{llll}\text { C } & 0.455719 & -0.172869 & -1.499076\end{array}$
$\begin{array}{llll}\text { C } & -0.878451 & 0.511722 & -1.658006\end{array}$
$\begin{array}{lllll}\text { C } & -0.871187 & 1.983380 & -1.335449\end{array}$
$\begin{array}{llll}\text { C } & -0.496937 & 2.456827 & -0.156659\end{array}$
$\begin{array}{llll}\text { C } & -0.021229 & 1.569779 & 0.964447\end{array}$
$\begin{array}{llll}\mathrm{H} & 0.237453 & 2.193451 & 1.817624\end{array}$
$\begin{array}{llll}\mathrm{C} & -1.108475 & 0.531264 & 1.456669\end{array}$
$\begin{array}{llll}\text { C } & -0.491466 & -0.750507 & 1.930747\end{array}$ 


$\begin{array}{cccc}\mathrm{C} & -0.565125 & -1.968667 & 1.386803 \\ \mathrm{C} & -1.182049 & -2.422937 & 0.142197 \\ \mathrm{C} & -1.770156 & -1.723132 & -0.831683 \\ \mathrm{C} & -2.014325 & -0.242919 & -0.865750 \\ \mathrm{C} & -2.294799 & 0.341010 & 0.518333 \\ \mathrm{H} & -2.785198 & 1.306242 & 0.391839 \\ \mathrm{H} & -3.015512 & -0.310429 & 1.017001 \\ \mathrm{H} & -2.926861 & -0.085762 & -1.447224 \\ \mathrm{H} & -2.113957 & -2.277967 & -1.699546 \\ \mathrm{H} & -1.102254 & -3.492884 & -0.019479 \\ \mathrm{H} & -0.055976 & -2.757613 & 1.930380 \\ \mathrm{H} & 0.084115 & -0.663055 & 2.847407 \\ \mathrm{H} & -1.531637 & 0.997529 & 2.351232 \\ \mathrm{H} & -0.528165 & 3.523306 & 0.030544 \\ \mathrm{H} & -1.223264 & 2.664512 & -2.101242 \\ \mathrm{H} & -1.126483 & 0.401551 & -2.714383 \\ \mathrm{H} & 0.669095 & -0.923106 & -2.251044 \\ \mathrm{Cl} & 2.846153 & -0.931774 & -0.650953\end{array}$

9a

$\begin{array}{ll}\text { E } & -1076.677654 \\ \text { H } & -1076.427268 \\ \text { G } & -1076.478426\end{array}$

Imag. Freq. $\quad 1.000$

Cartesian coordinates

$\begin{array}{lrrr}\mathrm{C} & 0.623047 & 0.183225 & 1.163125 \\ \mathrm{O} & 1.364127 & 0.217419 & 2.104016 \\ \mathrm{C} & -0.874909 & 0.346014 & 1.391691 \\ \mathrm{C} & -1.385282 & 1.680492 & 0.939333 \\ \mathrm{C} & -0.839991 & 2.543048 & 0.081442 \\ \mathrm{C} & 0.357789 & 2.421776 & -0.746511 \\ \mathrm{C} & 1.192112 & 1.398483 & -0.933376 \\ \mathrm{C} & 1.158805 & 0.046299 & -0.274907 \\ \mathrm{C} & 0.365376 & -1.027154 & -1.106907 \\ \mathrm{C} & -0.002070 & -2.188683 & -0.217983 \\ \mathrm{C} & -0.937954 & -2.115550 & 0.718493 \\ \mathrm{C} & -1.766802 & -0.878493 & 0.950184 \\ \mathrm{C} & -2.692333 & -0.608285 & -0.238802 \\ \mathrm{C} & -2.044088 & -0.283709 & -1.546736 \\ \mathrm{C} & -0.777009 & -0.435435 & -1.899511 \\ \mathrm{H} & -0.495139 & -0.095465 & -2.889769 \\ \mathrm{H} & -2.713564 & 0.143655 & -2.287113 \\ \mathrm{H} & -3.367463 & 0.214283 & 0.014176\end{array}$




$\begin{array}{cccc}\mathrm{H} & -3.337925 & -1.483772 & -0.372845 \\ \mathrm{H} & -2.424985 & -1.073792 & 1.797663 \\ \mathrm{H} & -1.127070 & -2.977439 & 1.348019 \\ \mathrm{H} & 0.570561 & -3.100583 & -0.333600 \\ \mathrm{H} & 1.082806 & -1.382621 & -1.844217 \\ \mathrm{Cl} & 2.863791 & -0.536587 & -0.163991 \\ \mathrm{H} & 1.997486 & 1.522884 & -1.646536 \\ \mathrm{H} & 0.570370 & 3.305030 & -1.338877 \\ \mathrm{H} & -1.373451 & 3.476946 & -0.058193 \\ \mathrm{H} & -2.317837 & 1.978652 & 1.407996 \\ \mathrm{H} & -0.921401 & 0.362672 & 2.481806\end{array}$

\section{$10 a$}

$\begin{array}{lr}\mathrm{E} & -1076.682432 \\ \mathrm{H} & -1076.432355 \\ \mathrm{G} & -1076.485228 \\ \text { Imag. Freq. } & 1.000\end{array}$

Cartesian coordinates

$\begin{array}{lrrr}\mathrm{C} & -0.937095 & -0.339877 & -0.302520 \\ \mathrm{C} & -2.277957 & -0.377528 & -0.208802 \\ \mathrm{C} & -3.102466 & 0.799806 & -0.200466 \\ \mathrm{C} & -2.749516 & 2.018392 & 0.272074 \\ \mathrm{C} & -1.494753 & 2.345384 & 0.906649 \\ \mathrm{C} & -0.309938 & 1.798250 & 0.604442 \\ \mathrm{C} & -0.170049 & 0.912984 & -0.602901 \\ \mathrm{H} & -0.623350 & 1.425229 & -1.456103 \\ \mathrm{C} & 1.213311 & 0.346475 & -0.972616 \\ \mathrm{H} & 1.192992 & 0.143120 & -2.046150 \\ \mathrm{C} & 2.432333 & 1.210228 & -0.662247 \\ \mathrm{C} & 3.740851 & 0.469708 & -0.735927 \\ \mathrm{C} & 4.097320 & -0.448540 & 0.164248 \\ \mathrm{C} & 3.209212 & -0.888108 & 1.241642 \\ \mathrm{C} & 1.902390 & -1.089450 & 1.072477 \\ \mathrm{C} & 1.233310 & -1.026127 & -0.272858 \\ \mathrm{H} & 1.709292 & -1.764685 & -0.922156 \\ \mathrm{O} & -0.142080 & -1.410211 & -0.161134 \\ \mathrm{H} & 1.279397 & -1.413176 & 1.898067 \\ \mathrm{H} & 3.650504 & -1.076171 & 2.214642 \\ \mathrm{H} & 5.092218 & -0.879448 & 0.124149 \\ \mathrm{H} & 4.441497 & 0.745789 & -1.515587 \\ \mathrm{H} & 2.340495 & 1.598980 & 0.354161 \\ \mathrm{H} & 2.442559 & 2.070085 & -1.332996 \\ \mathrm{H} & 0.550930 & 2.006867 & 1.226397\end{array}$




$\begin{array}{llll}\mathrm{H} & -1.539512 & 3.067272 & 1.715605 \\ \mathrm{H} & -3.522964 & 2.777673 & 0.296630 \\ \mathrm{H} & -4.131249 & 0.654228 & -0.510297 \\ \mathrm{Cl} & -3.086346 & -1.909627 & -0.033910\end{array}$

11a

$\begin{array}{lr}\mathrm{E} & -1076.715364 \\ \mathrm{H} & -1076.463179 \\ \mathrm{G} & -1076.510542 \\ \text { Imag. Freq. } & 1.000\end{array}$

Cartesian coordinates

$\begin{array}{lrrr}\mathrm{C} & -0.140360 & -1.726136 & 0.261105 \\ \mathrm{O} & -0.455018 & -2.852655 & 0.025604 \\ \mathrm{C} & -0.888312 & -0.450502 & -0.104403 \\ \mathrm{C} & -0.208417 & 0.322744 & -1.255077 \\ \mathrm{H} & -0.718241 & 0.053494 & -2.175714 \\ \mathrm{C} & 1.353903 & 0.343530 & -1.402040 \\ \mathrm{C} & 2.312040 & -0.742804 & -1.026850 \\ \mathrm{C} & 2.249411 & -1.423958 & 0.108275 \\ \mathrm{C} & 1.086181 & -1.269063 & 1.053155 \\ \mathrm{C} & 0.745758 & 0.176903 & 1.540914 \\ \mathrm{H} & 0.891446 & 0.219369 & 2.620247 \\ \mathrm{C} & 1.582326 & 1.320812 & 0.943518 \\ \mathrm{C} & 1.371781 & 1.624834 & -0.537177 \\ \mathrm{H} & 2.071510 & 2.401976 & -0.847360 \\ \mathrm{C} & -0.113261 & 1.858790 & -0.948557 \\ \mathrm{C} & -1.028342 & 2.427020 & 0.076756 \\ \mathrm{C} & -1.319062 & 1.697263 & 1.144275 \\ \mathrm{C} & -0.773641 & 0.307810 & 1.235593 \\ \mathrm{H} & -1.325963 & -0.263272 & 1.985242 \\ \mathrm{H} & -1.973962 & 2.060647 & 1.925434 \\ \mathrm{H} & -1.426580 & 3.425927 & -0.052185 \\ \mathrm{H} & -0.149427 & 2.422137 & -1.880105 \\ \mathrm{H} & 2.638944 & 1.094743 & 1.101787 \\ \mathrm{H} & 1.369489 & 2.225414 & 1.516683 \\ \mathrm{H} & 2.998918 & -2.169007 & 0.342309 \\ \mathrm{H} & 3.145557 & -0.914126 & -1.698622 \\ \mathrm{H} & 1.531103 & 0.624080 & -2.440756 \\ \mathrm{H} & 1.204328 & -1.940507 & 1.902196 \\ \mathrm{Cl} & -2.588060 & -0.777829 & -0.531818\end{array}$

$12 a$ 


$\begin{array}{lr}\mathrm{E} & -1076.714907 \\ \mathrm{H} & -1076.462547 \\ \mathrm{G} & -1076.510235 \\ \text { Imag. Freq. } & 1.000\end{array}$

Cartesian coordinates

$\begin{array}{lrrr}\mathrm{C} & 0.529378 & -0.146045 & 1.181057 \\ \mathrm{C} & -0.985559 & 0.046137 & 1.297811 \\ \mathrm{C} & 1.175856 & -0.068959 & -0.214002 \\ \mathrm{C} & -1.477435 & 1.313227 & 0.554781 \\ \mathrm{C} & 0.778810 & 1.202786 & -1.018944 \\ \mathrm{C} & -0.548710 & 2.485815 & 0.540254 \\ \mathrm{C} & 0.519223 & 2.445624 & -0.245179 \\ \mathrm{H} & -2.430553 & 1.603539 & 1.001442 \\ \mathrm{H} & -0.773522 & 3.352526 & 1.149138 \\ \mathrm{H} & 1.216098 & 3.271465 & -0.309540 \\ \mathrm{H} & 1.523008 & 1.347304 & -1.799111 \\ \mathrm{H} & -1.153310 & 0.122219 & 2.369939 \\ \mathrm{O} & 1.181411 & -0.352384 & 2.165547 \\ \mathrm{C} & -2.617612 & -0.460602 & -0.500376 \\ \mathrm{C} & -1.866890 & -1.113748 & 0.691112 \\ \mathrm{C} & -1.754786 & 0.744173 & -0.847163 \\ \mathrm{C} & -1.101210 & -2.315635 & 0.212501 \\ \mathrm{C} & -0.445106 & 0.390556 & -1.570070 \\ \mathrm{C} & -0.155909 & -2.218868 & -0.710833 \\ \mathrm{C} & 0.362952 & -0.912522 & -1.222847 \\ \mathrm{H} & -1.375626 & -3.288017 & 0.603990 \\ \mathrm{H} & 0.353940 & -3.111054 & -1.054456 \\ \mathrm{H} & 1.010589 & -1.091129 & -2.079958 \\ \mathrm{H} & -0.597528 & 0.492757 & -2.642539 \\ \mathrm{H} & -2.288007 & 1.478422 & -1.452903 \\ \mathrm{H} & -2.575040 & -1.427445 & 1.455759 \\ \mathrm{H} & -2.750234 & -1.159688 & -1.324582 \\ \mathrm{H} & -3.606782 & -0.117023 & -0.191048 \\ \mathrm{Cl} & 2.912803 & -0.412143 & -0.114065\end{array}$

\section{TS-5a}

$\begin{array}{lr}\mathrm{E} & -1076.625049 \\ \mathrm{H} & -1076.378237 \\ \mathrm{G} & -1076.431841 \\ \text { Imag. Freq. } & -444.362\end{array}$

Cartesian coordinates 


$\begin{array}{lccc}\mathrm{C} & -0.752350 & 0.063085 & -1.190458 \\ \mathrm{C} & 0.637550 & 0.476070 & -1.564432 \\ \mathrm{C} & -1.434667 & 0.598444 & -0.024721 \\ \mathrm{C} & 1.223928 & 1.746582 & -1.292438 \\ \mathrm{C} & -1.006519 & 1.551123 & 0.861751 \\ \mathrm{C} & 1.058038 & 2.528992 & -0.179840 \\ \mathrm{C} & 0.137104 & 2.351916 & 0.849803 \\ \mathrm{H} & 1.980908 & 2.067063 & -2.000672 \\ \mathrm{H} & 1.722696 & 3.380953 & -0.082189 \\ \mathrm{H} & 0.224681 & 3.031189 & 1.689501 \\ \mathrm{H} & -1.670028 & 1.714239 & 1.703262 \\ \mathrm{H} & 0.757309 & 0.176957 & -2.599843 \\ \mathrm{O} & -1.259550 & -0.802278 & -1.885013 \\ \mathrm{C} & 2.530809 & -0.285176 & 0.373794 \\ \mathrm{C} & 1.834252 & -0.833116 & -0.860054 \\ \mathrm{C} & 1.749771 & -0.113452 & 1.634413 \\ \mathrm{C} & 1.078899 & -2.020695 & -0.843270 \\ \mathrm{C} & 0.672192 & -0.802963 & 2.044410 \\ \mathrm{C} & 0.224189 & -2.454391 & 0.166515 \\ \mathrm{C} & -0.019103 & -1.858218 & 1.381172 \\ \mathrm{H} & 1.027664 & -2.571823 & -1.774550 \\ \mathrm{H} & -0.397568 & -3.307227 & -0.077085 \\ \mathrm{H} & -0.828659 & -2.294675 & 1.955879 \\ \mathrm{H} & 0.265151 & -0.531038 & 3.012178 \\ \mathrm{H} & 2.159940 & 0.615186 & 2.325062 \\ \mathrm{H} & 2.522910 & -0.797016 & -1.699659 \\ \mathrm{H} & 3.406151 & -0.912237 & 0.584898 \\ \mathrm{H} & 2.941996 & 0.693122 & 0.101093 \\ \mathrm{Cl} & -3.037724 & -0.031510 & 0.223668\end{array}$

\section{TS-S4a}

$\begin{array}{lr}\mathrm{E} & -1076.619542 \\ \mathrm{H} & -1076.373144 \\ \mathrm{G} & -1076.425982 \\ \text { Imag. Freq. } & -465.269\end{array}$

Cartesian coordinates
$\begin{array}{llll}\text { C } & -0.684366 & -0.049072 & 1.285766\end{array}$
$\begin{array}{llll}\text { C } & -1.197266 & 0.366085 & -0.079875\end{array}$
$\begin{array}{llll}\text { C } & 0.506034 & 0.521773 & 1.873570\end{array}$
$\begin{array}{llll}\text { C } & -1.004666 & 1.671234 & -0.631377\end{array}$
$\begin{array}{llll}\text { C } & 1.290699 & 1.566071 & 1.471045\end{array}$
$\begin{array}{llll}\text { C } & 0.059715 & 2.522116 & -0.481068\end{array}$
$\begin{array}{llll}\text { C } & 1.149806 & 2.422589 & 0.379744\end{array}$ 


$\begin{array}{lrcc}\mathrm{H} & -1.755442 & 1.966360 & -1.353727 \\ \mathrm{H} & 0.051426 & 3.389065 & -1.133437 \\ \mathrm{H} & 1.912074 & 3.185380 & 0.273404 \\ \mathrm{H} & 2.164116 & 1.748730 & 2.089002 \\ \mathrm{O} & -1.242225 & -0.974250 & 1.847571 \\ \mathrm{C} & 0.789045 & -0.180643 & -2.032077 \\ \mathrm{C} & -0.360833 & -0.856755 & -1.307009 \\ \mathrm{C} & 2.083215 & 0.056314 & -1.329613 \\ \mathrm{C} & -0.206508 & -2.049706 & -0.568921 \\ \mathrm{C} & 2.595131 & -0.603982 & -0.278576 \\ \mathrm{C} & 0.860952 & -2.387959 & 0.252460 \\ \mathrm{C} & 2.029332 & -1.685556 & 0.458480 \\ \mathrm{H} & -1.079220 & -2.687501 & -0.504443 \\ \mathrm{H} & 0.715005 & -3.259098 & 0.879774 \\ \mathrm{H} & 2.670654 & -2.077731 & 1.240597 \\ \mathrm{H} & 3.568258 & -0.276111 & 0.070194 \\ \mathrm{H} & 2.707701 & 0.817774 & -1.784377 \\ \mathrm{H} & -1.219387 & -0.894108 & -1.968890 \\ \mathrm{H} & 0.991073 & -0.754543 & -2.945282 \\ \mathrm{H} & 0.418484 & 0.786776 & -2.389560 \\ \mathrm{H} & 0.757071 & 0.027759 & 2.804045 \\ \mathrm{Cl} & -2.907398 & -0.120812 & -0.243127\end{array}$

\section{TS-6a}

$\begin{array}{lr}\text { E } & -1076.628631 \\ \mathrm{H} & -1076.381322 \\ \mathrm{G} & -1076.433059 \\ \text { Imag. Freq. } & -94.713\end{array}$

Cartesian coordinates

$\begin{array}{lrrr}\mathrm{C} & -0.704090 & -0.028939 & -1.186445 \\ \mathrm{C} & 0.768897 & 0.205416 & -1.510950 \\ \mathrm{C} & -1.365731 & 0.572683 & -0.039666 \\ \mathrm{C} & 1.350539 & 1.556571 & -1.388225 \\ \mathrm{C} & -0.908407 & 1.596525 & 0.772301 \\ \mathrm{C} & 1.177809 & 2.428088 & -0.378239 \\ \mathrm{C} & 0.261412 & 2.323256 & 0.706469 \\ \mathrm{H} & 2.072924 & 1.818419 & -2.154272 \\ \mathrm{H} & 1.798446 & 3.318323 & -0.380096 \\ \mathrm{H} & 0.388770 & 3.056226 & 1.494706 \\ \mathrm{H} & -1.558217 & 1.833194 & 1.607189 \\ \mathrm{H} & 0.806395 & -0.092845 & -2.556884 \\ \mathrm{O} & -1.278173 & -0.825005 & -1.906583 \\ \mathrm{C} & 2.486521 & -0.369817 & 0.446732\end{array}$




$\begin{array}{lccc}\mathrm{C} & 1.751118 & -0.892895 & -0.792683 \\ \mathrm{C} & 1.711514 & -0.035940 & 1.673555 \\ \mathrm{C} & 1.014699 & -2.153222 & -0.635835 \\ \mathrm{C} & 0.546644 & -0.588124 & 2.077861 \\ \mathrm{C} & 0.098588 & -2.397224 & 0.332307 \\ \mathrm{C} & -0.235456 & -1.553753 & 1.415092 \\ \mathrm{H} & 1.086643 & -2.868992 & -1.445928 \\ \mathrm{H} & -0.522467 & -3.277260 & 0.214532 \\ \mathrm{H} & -1.153066 & -1.820557 & 1.927486 \\ \mathrm{H} & 0.121911 & -0.205675 & 2.998974 \\ \mathrm{H} & 2.195128 & 0.658466 & 2.351492 \\ \mathrm{H} & 2.529053 & -1.037218 & -1.543273 \\ \mathrm{H} & 3.259188 & -1.098476 & 0.722237 \\ \mathrm{H} & 3.034309 & 0.526670 & 0.139843 \\ \mathrm{Cl} & -3.032822 & 0.106707 & 0.171942\end{array}$

\section{TS-S5a}

$\begin{array}{lr}\mathrm{E} & -1076.623724 \\ \mathrm{H} & -1076.377045 \\ \mathrm{G} & -1076.428356 \\ \text { Imag. Freq. } & -111.622\end{array}$

Cartesian coordinates

$\begin{array}{lrrc}\mathrm{C} & -0.653381 & -0.077100 & 1.285965 \\ \mathrm{C} & -1.114361 & 0.349506 & -0.131409 \\ \mathrm{C} & 0.599737 & 0.360090 & 1.865439 \\ \mathrm{C} & -0.898538 & 1.753288 & -0.550939 \\ \mathrm{C} & 1.457893 & 1.355645 & 1.467456 \\ \mathrm{C} & 0.206900 & 2.502878 & -0.381979 \\ \mathrm{C} & 1.367321 & 2.216487 & 0.387756 \\ \mathrm{H} & -1.686732 & 2.159007 & -1.171708 \\ \mathrm{H} & 0.223004 & 3.448290 & -0.914676 \\ \mathrm{H} & 2.192998 & 2.906875 & 0.260601 \\ \mathrm{H} & 2.369985 & 1.436775 & 2.050545 \\ \mathrm{O} & -1.318735 & -0.908916 & 1.867579 \\ \mathrm{C} & 0.740482 & -0.115360 & -2.003285 \\ \mathrm{C} & -0.483620 & -0.661450 & -1.253790 \\ \mathrm{C} & 2.043499 & 0.054607 & -1.303372 \\ \mathrm{C} & -0.354703 & -2.010471 & -0.679339 \\ \mathrm{C} & 2.520855 & -0.706455 & -0.287561 \\ \mathrm{C} & 0.677034 & -2.438538 & 0.083827 \\ \mathrm{C} & 1.866194 & -1.736632 & 0.403164 \\ \mathrm{H} & -1.222982 & -2.652857 & -0.755356 \\ \mathrm{H} & 0.551742 & -3.390429 & 0.586808\end{array}$




$\begin{array}{cccc}\mathrm{H} & 2.451773 & -2.189765 & 1.195970 \\ \mathrm{H} & 3.506038 & -0.451857 & 0.086661 \\ \mathrm{H} & 2.724976 & 0.763102 & -1.759606 \\ \mathrm{H} & -1.277326 & -0.680362 & -1.998318 \\ \mathrm{H} & 0.912476 & -0.776800 & -2.861630 \\ \mathrm{H} & 0.452963 & 0.845799 & -2.437356 \\ \mathrm{Cl} & -2.900405 & 0.054607 & -0.201757 \\ \mathrm{H} & 0.805977 & -0.153737 & 2.795704\end{array}$

\section{TS-7a \\ E $\quad-1076.623270$ \\ $\mathrm{H} \quad-1076.375810$ \\ G $\quad-1076.428141$ \\ Imag. Freq. $\quad-230.068$}

Cartesian coordinates

$\begin{array}{lrrr}\mathrm{C} & -0.627545 & -0.020236 & -1.091907 \\ \mathrm{C} & 0.759217 & 0.476104 & -1.445140 \\ \mathrm{C} & -1.474850 & 0.532589 & -0.126060 \\ \mathrm{C} & 1.226534 & 1.849615 & -1.178648 \\ \mathrm{C} & -1.238622 & 1.627271 & 0.723963 \\ \mathrm{C} & 0.855410 & 2.680432 & -0.190958 \\ \mathrm{C} & -0.196871 & 2.507012 & 0.759728 \\ \mathrm{H} & 2.011333 & 2.196175 & -1.844241 \\ \mathrm{H} & 1.392908 & 3.620640 & -0.116921 \\ \mathrm{H} & -0.245127 & 3.254491 & 1.543019 \\ \mathrm{H} & -2.000296 & 1.767255 & 1.482341 \\ \mathrm{H} & 0.799335 & 0.309535 & -2.522106 \\ \mathrm{O} & -0.859362 & -1.105336 & -1.667906 \\ \mathrm{C} & 2.516282 & -0.264608 & 0.392824 \\ \mathrm{C} & 1.772392 & -0.669102 & -0.883195 \\ \mathrm{C} & 1.710030 & 0.036224 & 1.612743 \\ \mathrm{C} & 1.034058 & -1.943488 & -0.844211 \\ \mathrm{C} & 0.666242 & -0.676442 & 2.052647 \\ \mathrm{C} & 0.377799 & -2.479318 & 0.266828 \\ \mathrm{C} & 0.115345 & -1.859826 & 1.462537 \\ \mathrm{H} & 1.096332 & -2.572282 & -1.721019 \\ \mathrm{H} & -0.122545 & -3.425072 & 0.099983 \\ \mathrm{H} & -0.598600 & -2.377917 & 2.094744 \\ \mathrm{H} & 0.190937 & -0.355214 & 2.972068 \\ \mathrm{H} & 2.037283 & 0.876468 & 2.213517 \\ \mathrm{H} & 2.534429 & -0.749552 & -1.659728 \\ \mathrm{H} & 3.234900 & -1.056505 & 0.640632 \\ \mathrm{H} & 3.114485 & 0.616110 & 0.148016 \\ \mathrm{Cl} & -3.031941 & -0.237577 & 0.055649\end{array}$




\section{TS-8a}

$$
\begin{array}{lr}
\mathrm{E} & -1076.631418 \\
\mathrm{H} & -1076.382769 \\
\mathrm{G} & -1076.431395 \\
\text { Imag. Freq. } & -654.777
\end{array}
$$

Cartesian coordinates

$\begin{array}{lrrr}\mathrm{C} & 0.547113 & -0.089520 & 1.184426 \\ \mathrm{C} & -0.966080 & 0.012655 & 1.310456 \\ \mathrm{C} & 1.159733 & 0.048861 & -0.218402 \\ \mathrm{C} & -1.536009 & 1.301556 & 0.756081 \\ \mathrm{C} & 0.978581 & 1.445445 & -0.786720 \\ \mathrm{C} & -0.840652 & 2.472646 & 0.493583 \\ \mathrm{C} & 0.342204 & 2.533338 & -0.233957 \\ \mathrm{H} & -2.553944 & 1.457333 & 1.103779 \\ \mathrm{H} & -1.382575 & 3.403519 & 0.614916 \\ \mathrm{H} & 0.627628 & 3.506171 & -0.618607 \\ \mathrm{H} & 1.698668 & 1.647061 & -1.572337 \\ \mathrm{H} & -1.118306 & 0.032893 & 2.390350 \\ \mathrm{O} & 1.220195 & -0.263187 & 2.159315 \\ \mathrm{C} & -2.625479 & -0.669181 & -0.405609 \\ \mathrm{C} & -1.750877 & -1.218224 & 0.727308 \\ \mathrm{C} & -1.860767 & 0.413075 & -1.135479 \\ \mathrm{C} & -0.880920 & -2.336782 & 0.216740 \\ \mathrm{C} & -0.615123 & 0.203964 & -1.707968 \\ \mathrm{C} & 0.040163 & -2.165594 & -0.721499 \\ \mathrm{C} & 0.430092 & -0.819524 & -1.261152 \\ \mathrm{H} & -1.053077 & -3.329100 & 0.617047 \\ \mathrm{H} & 0.622676 & -3.014509 & -1.058335 \\ \mathrm{H} & 1.117647 & -0.955248 & -2.094295 \\ \mathrm{H} & -0.411538 & 0.678045 & -2.656961 \\ \mathrm{H} & -2.474905 & 1.153954 & -1.635782 \\ \mathrm{H} & -2.387111 & -1.614127 & 1.517688 \\ \mathrm{H} & -2.912322 & -1.471131 & -1.088398 \\ \mathrm{H} & -3.547450 & -0.246584 & -0.004122 \\ \mathrm{Cl} & 2.910657 & -0.349357 & -0.123312\end{array}$

\section{TS-9a}

$\begin{array}{ll}\text { E } & -1076.642313 \\ \text { H } & -1076.393161 \\ \text { G } & -1076.441737 \\ \text { Imag. Freq. } & -645.620\end{array}$


Cartesian coordinates

$\begin{array}{lrrr}\mathrm{C} & -0.701978 & -1.577666 & 0.290629 \\ \mathrm{C} & 0.688328 & -1.569641 & 0.918773 \\ \mathrm{C} & -1.009180 & -0.317486 & -0.432347 \\ \mathrm{C} & 1.690250 & -2.009439 & -0.113179 \\ \mathrm{C} & -0.150517 & 0.293417 & -1.347056 \\ \mathrm{C} & 1.948501 & -1.301735 & -1.203783 \\ \mathrm{C} & 1.358492 & 0.050340 & -1.470855 \\ \mathrm{H} & 2.179844 & -2.963302 & 0.039214 \\ \mathrm{H} & 2.672650 & -1.675816 & -1.918418 \\ \mathrm{H} & 1.625747 & 0.351887 & -2.483780 \\ \mathrm{H} & -0.638972 & 0.665398 & -2.237254 \\ \mathrm{H} & 0.650433 & -2.308136 & 1.717163 \\ \mathrm{O} & -1.483669 & -2.478604 & 0.433346 \\ \mathrm{C} & 1.999726 & 0.708037 & 0.928182 \\ \mathrm{C} & 0.889128 & -0.145539 & 1.532740 \\ \mathrm{C} & 1.788325 & 1.171112 & -0.503395 \\ \mathrm{C} & -0.466413 & 0.529353 & 1.582757 \\ \mathrm{C} & 0.586743 & 2.088936 & -0.700109 \\ \mathrm{C} & -0.779786 & 1.852492 & 1.327512 \\ \mathrm{C} & -0.283772 & 2.586656 & 0.259853 \\ \mathrm{H} & -1.152584 & 0.021504 & 2.257896 \\ \mathrm{H} & -1.658664 & 2.250658 & 1.820740 \\ \mathrm{H} & -0.811748 & 3.502146 & 0.016855 \\ \mathrm{H} & 0.675122 & 2.697463 & -1.596666 \\ \mathrm{H} & 2.701128 & 1.676983 & -0.829277 \\ \mathrm{H} & 1.175474 & -0.298032 & 2.577517 \\ \mathrm{H} & 2.133141 & 1.590600 & 1.557562 \\ \mathrm{H} & 2.932701 & 0.143065 & 0.976146 \\ \mathrm{Cl} & -2.703646 & 0.001258 & -0.693106\end{array}$




\section{Cycloheptatriene and 2-methoxytropone calculated structures}

\section{$1 \mathbf{b}$}

E

$\mathrm{H}$

G

Imag. Freq. 1.000

Cartesian coordinates

$\begin{array}{llll}\mathrm{H} & -2.208209 & -1.989880 & 0.000000 \\ \mathrm{H} & -0.467793 & -3.507190 & 0.000000 \\ \mathrm{H} & 1.852691 & -3.110062 & 0.000000 \\ \mathrm{H} & 2.930257 & -1.050931 & 0.000000 \\ \mathrm{H} & 2.045554 & 1.063423 & 0.000000 \\ \mathrm{H} & 1.291935 & 2.924769 & -0.893411 \\ \mathrm{H} & 1.291935 & 2.924769 & 0.893411 \\ \mathrm{H} & 0.132469 & 3.936849 & 0.000000 \\ \mathrm{C} & 0.663600 & 2.988613 & 0.000000 \\ \mathrm{O} & -0.332607 & 1.993419 & 0.000000 \\ \mathrm{C} & 0.000000 & 0.702801 & 0.000000 \\ \mathrm{C} & 1.295841 & 0.283337 & 0.000000 \\ \mathrm{C} & 1.845975 & -1.027464 & 0.000000 \\ \mathrm{C} & 1.223254 & -2.228454 & 0.000000 \\ \mathrm{C} & -0.178751 & -2.460951 & 0.000000 \\ \mathrm{C} & -1.204016 & -1.580627 & 0.000000 \\ \mathrm{C} & -1.248057 & -0.124107 & 0.000000 \\ \mathrm{O} & -2.324383 & 0.442753 & 0.000000\end{array}$

\section{$\mathbf{8 b}$}

$\begin{array}{ll}\text { E } & -731.313579 \\ H & -731.312635 \\ \text { G } & -731.366828\end{array}$

Imag. Freq. $\quad 1.000$

Cartesian coordinates

$\begin{array}{llll}\mathrm{H} & -3.188867 & 0.749311 & 0.669942 \\ \mathrm{H} & -4.107916 & 1.223350 & -0.776452 \\ \mathrm{H} & -2.518630 & 1.964451 & -0.455781 \\ \mathrm{C} & -3.111164 & 1.046851 & -0.377004 \\ \mathrm{O} & -2.539394 & 0.021453 & -1.170124 \\ \mathrm{H} & 2.899749 & -0.761828 & 0.784863 \\ \mathrm{H} & 2.749324 & 0.912590 & 1.263302\end{array}$




$\begin{array}{llll}\mathrm{H} & 1.366121 & -0.554801 & 2.544223 \\ \mathrm{H} & 3.027602 & 0.485051 & -1.156440 \\ \mathrm{H} & 1.859147 & 2.443186 & -1.730244 \\ \mathrm{H} & 0.458212 & 3.580857 & -0.294803 \\ \mathrm{H} & -0.660633 & 2.828188 & 1.607655 \\ \mathrm{H} & -0.554552 & 0.817763 & 2.702404 \\ \mathrm{C} & 0.705628 & 2.561142 & -0.017600 \\ \mathrm{C} & 0.033616 & 2.102031 & 1.196544 \\ \mathrm{C} & 1.520572 & 1.906393 & -0.848975 \\ \mathrm{C} & 0.101995 & 0.935740 & 1.845333 \\ \mathrm{C} & 2.031672 & 0.504113 & -0.704971 \\ \mathrm{C} & 0.985476 & -0.242908 & 1.567069 \\ \mathrm{C} & 2.230000 & 0.097416 & 0.754843 \\ \mathrm{O} & -2.196506 & -1.452250 & 1.073294 \\ \mathrm{H} & -0.089912 & -2.089042 & 1.933215 \\ \mathrm{H} & -0.540465 & 0.487493 & -2.492074 \\ \mathrm{H} & 1.547412 & -0.451745 & -2.567134 \\ \mathrm{H} & 1.962072 & -2.594341 & -1.706491 \\ \mathrm{H} & 1.146709 & -3.389345 & 0.406682 \\ \mathrm{C} & 1.171817 & -0.518708 & -1.545401 \\ \mathrm{C} & 1.390846 & -1.931551 & -1.066936 \\ \mathrm{C} & -0.276252 & -0.112593 & -1.628043 \\ \mathrm{C} & 0.949144 & -2.368442 & 0.102861 \\ \mathrm{C} & -1.288215 & -0.377658 & -0.804782 \\ \mathrm{C} & 0.171046 & -1.499504 & 1.056443 \\ \mathrm{C} & -1.194207 & -1.136449 & 0.477580\end{array}$

9b

$\begin{array}{ll}\text { E } & -731.304756 \\ \text { H } & -731.303812 \\ \text { G } & -731.357167 \\ \text { Imag. Freq. } & 1.000\end{array}$

Cartesian coordinates

$\begin{array}{llll}\mathrm{H} & 2.937689 & -0.974964 & 1.000398 \\ \mathrm{H} & 4.287353 & -0.279852 & 0.086054 \\ \mathrm{H} & 3.214890 & -1.451797 & -0.697546 \\ \mathrm{C} & 3.256203 & -0.622445 & 0.018350 \\ \mathrm{O} & 2.507962 & 0.484710 & -0.425469 \\ \mathrm{H} & -3.288265 & -0.561145 & 0.156362 \\ \mathrm{H} & -2.870259 & -2.202308 & -0.252498 \\ \mathrm{H} & -1.975840 & -1.601921 & 1.871662 \\ \mathrm{H} & -2.763225 & -0.478992 & -2.176132 \\ \mathrm{H} & -0.592445 & -0.163734 & -2.902173\end{array}$




$\begin{array}{llll}\mathrm{H} & 1.324217 & -0.983020 & -1.935339 \\ \mathrm{H} & 1.274975 & -2.826074 & -0.479327 \\ \mathrm{H} & -0.290435 & -3.136274 & 1.293272 \\ \mathrm{C} & 0.564459 & -0.833362 & -1.167170 \\ \mathrm{C} & 0.527066 & -2.063830 & -0.295345 \\ \mathrm{C} & -0.725285 & -0.560715 & -1.901962 \\ \mathrm{C} & -0.340022 & -2.234738 & 0.693318 \\ \mathrm{C} & -1.969190 & -0.725951 & -1.477871 \\ \mathrm{C} & -1.427630 & -1.239904 & 1.000723 \\ \mathrm{C} & -2.449029 & -1.196507 & -0.140276 \\ \mathrm{O} & 1.400152 & 0.610979 & 1.989955 \\ \mathrm{H} & -0.813716 & 0.140438 & 2.523613 \\ \mathrm{H} & 1.388376 & 2.065293 & -1.738318 \\ \mathrm{H} & -0.458897 & 3.394590 & -1.330844 \\ \mathrm{H} & -2.279252 & 3.042189 & 0.094687 \\ \mathrm{H} & -2.657543 & 1.354848 & 1.613357 \\ \mathrm{C} & -0.385900 & 2.486333 & -0.742505 \\ \mathrm{C} & -1.505784 & 2.285789 & 0.173889 \\ \mathrm{C} & 0.677583 & 1.725076 & -0.993965 \\ \mathrm{C} & -1.723480 & 1.315891 & 1.061518 \\ \mathrm{C} & 1.103884 & 0.421989 & -0.365719 \\ \mathrm{C} & -0.843651 & 0.159942 & 1.432732 \\ \mathrm{C} & 0.623687 & 0.398633 & 1.097096\end{array}$

\section{0b}

$\begin{array}{ll}\text { E } & -731.305814 \\ \text { H } & -731.304870 \\ \text { G } & -731.361166 \\ \text { Imag. Freq. } & 1.000\end{array}$

Cartesian coordinates

$\begin{array}{llll}\mathrm{C} & 3.197706 & -0.668624 & 1.322121 \\ \mathrm{C} & 4.053414 & -0.434752 & 0.157583 \\ \mathrm{C} & 1.883972 & -0.87504 & 1.229067 \\ \mathrm{C} & 1.173598 & -1.028876 & -0.087853 \\ \mathrm{C} & 3.676934 & 0.320309 & -0.876403 \\ \mathrm{C} & 2.382305 & 1.089086 & -0.892716 \\ \mathrm{C} & 1.135853 & 0.21403 & -0.995125 \\ \mathrm{C} & -0.216357 & 0.861527 & -0.647511 \\ \mathrm{C} & -0.993469 & -0.318373 & -0.150395 \\ \mathrm{C} & -2.335485 & -0.330461 & -0.009156 \\ \mathrm{C} & -3.11658 & 0.873913 & -0.108978 \\ \mathrm{C} & -0.276451 & 1.895628 & 0.441411 \\ \mathrm{C} & -1.434282 & 2.511361 & 0.719798\end{array}$




$\begin{array}{llll}\mathrm{C} & -2.722727 & 2.134979 & 0.193475 \\ \mathrm{O} & -0.196426 & -1.380319 & 0.121232 \\ \mathrm{H} & 3.669296 & -0.693707 & 2.298817 \\ \mathrm{H} & 5.041651 & -0.882791 & 0.160489 \\ \mathrm{H} & 1.283137 & -1.039568 & 2.115965 \\ \mathrm{H} & 1.63407 & -1.862768 & -0.623798 \\ \mathrm{H} & 4.355184 & 0.44467 & -1.712934 \\ \mathrm{H} & 2.379893 & 1.811708 & -1.709534 \\ \mathrm{H} & 2.336904 & 1.661068 & 0.036384 \\ \mathrm{H} & 1.051564 & -0.157922 & -2.019663 \\ \mathrm{H} & -0.691181 & 1.276249 & -1.541007 \\ \mathrm{O} & -3.060411 & -1.447482 & 0.310092 \\ \mathrm{H} & -4.165738 & 0.709459 & -0.331362 \\ \mathrm{H} & 0.615663 & 2.153218 & 0.998334 \\ \mathrm{H} & -1.426118 & 3.332261 & 1.429608 \\ \mathrm{H} & -3.484384 & 2.906384 & 0.170622 \\ \mathrm{C} & -2.794192 & -2.619641 & -0.435476 \\ \mathrm{H} & -3.618188 & -3.303307 & -0.236663 \\ \mathrm{H} & -1.85332 & -3.08541 & -0.137797 \\ \mathrm{H} & -2.759167 & -2.397533 & -1.507106\end{array}$

\section{1b}

$\begin{array}{ll}\text { E } & -731.340023 \\ \mathrm{H} & -731.339079 \\ \mathrm{G} & -731.388645 \\ \text { Imag. Freq. } & 1.000\end{array}$

Cartesian coordinates

$\begin{array}{llll}\mathrm{H} & -4.151331 & -0.329611 & -0.846204 \\ \mathrm{H} & -3.481471 & 0.500281 & 0.570788 \\ \mathrm{H} & -3.258271 & -1.262968 & 0.366360 \\ \mathrm{C} & -3.311080 & -0.303325 & -0.154901 \\ \mathrm{O} & -2.165455 & -0.060359 & -0.935491 \\ \mathrm{H} & 2.698500 & 0.397856 & 1.407651 \\ \mathrm{H} & 1.704095 & 1.804874 & 1.711802 \\ \mathrm{H} & 0.609887 & -0.033721 & 2.651071 \\ \mathrm{H} & 2.736666 & 1.833999 & -0.534378 \\ \mathrm{H} & 0.743964 & 2.418128 & -1.836871 \\ \mathrm{H} & -0.484909 & 3.688431 & -0.146782 \\ \mathrm{H} & -1.607903 & 2.484600 & 1.685082 \\ \mathrm{H} & -1.544944 & 0.073833 & 1.747520 \\ \mathrm{C} & -0.363349 & 2.620492 & -0.011485 \\ \mathrm{C} & -0.962792 & 1.974949 & 0.979873 \\ \mathrm{C} & 0.516783 & 1.853894 & -0.932940\end{array}$




$\begin{array}{llll}\mathrm{C} & -0.788122 & 0.495016 & 1.080992 \\ \mathrm{C} & 1.831945 & 1.252366 & -0.351290 \\ \mathrm{C} & 0.595811 & -0.020542 & 1.561116 \\ \mathrm{C} & 1.761401 & 0.883930 & 1.127586 \\ \mathrm{O} & -1.132439 & -2.619852 & -0.212731 \\ \mathrm{H} & 0.464986 & -2.189798 & 1.881918 \\ \mathrm{H} & -0.365848 & 0.270135 & -2.288734 \\ \mathrm{H} & 1.989215 & 0.268533 & -2.264609 \\ \mathrm{H} & 3.056317 & -1.639960 & -1.398712 \\ \mathrm{H} & 2.327744 & -2.847726 & 0.541797 \\ \mathrm{C} & 1.611350 & 0.027282 & -1.270487 \\ \mathrm{C} & 2.209973 & -1.271500 & -0.829804 \\ \mathrm{C} & 0.090997 & 0.393775 & -1.309755 \\ \mathrm{C} & 1.826740 & -1.932379 & 0.253623 \\ \mathrm{C} & -0.925673 & -0.191016 & -0.306723 \\ \mathrm{C} & 0.626110 & -1.501230 & 1.053606 \\ \mathrm{C} & -0.562351 & -1.614243 & 0.100269\end{array}$

$12 b$

$\begin{array}{ll}\text { E } & -731.340903 \\ \text { H } & -731.339959 \\ \text { G } & -731.389907 \\ \text { Imag. Freq. } & 1.000\end{array}$

Cartesian coordinates

$\begin{array}{llll}\mathrm{H} & -2.256761 & -1.953890 & -1.248013 \\ \mathrm{H} & 3.142784 & -0.840926 & 0.967367 \\ \mathrm{H} & 3.275732 & -1.131077 & -0.788959 \\ \mathrm{H} & 4.353493 & 0.034655 & 0.005296 \\ \mathrm{C} & 3.344794 & -0.374238 & 0.001784 \\ \mathrm{H} & -1.922253 & -2.113408 & 1.524577 \\ \mathrm{H} & -0.206188 & -3.481633 & 0.623803 \\ \mathrm{C} & -0.276768 & -2.473644 & 0.232265 \\ \mathrm{H} & 1.300611 & -2.764283 & -1.099430 \\ \mathrm{C} & 0.547409 & -2.078409 & -0.727725 \\ \mathrm{H} & -2.701426 & 0.685774 & -1.400099 \\ \mathrm{H} & -3.378741 & -1.238115 & -0.092464 \\ \mathrm{C} & -1.937154 & 0.179417 & -0.808454 \\ \mathrm{C} & -2.340596 & -1.238623 & -0.431270 \\ \mathrm{H} & 1.250041 & -0.637010 & -2.123070 \\ \mathrm{C} & 0.599566 & -0.677771 & -1.248515 \\ \mathrm{H} & -0.816286 & 0.313832 & -2.637050 \\ \mathrm{C} & -0.603969 & 0.277893 & -1.570432 \\ \mathrm{C} & -1.381814 & -1.588972 & 0.738592\end{array}$




$\begin{array}{llll}\mathrm{O} & 2.475626 & 0.707035 & -0.253226 \\ \mathrm{C} & 1.124345 & 0.409991 & -0.264422 \\ \mathrm{H} & -1.076912 & -0.164031 & 2.393091 \\ \mathrm{C} & -0.923175 & -0.193885 & 1.316166 \\ \mathrm{O} & 1.272445 & 0.211337 & 2.109777 \\ \mathrm{C} & 0.558364 & 0.143502 & 1.143928 \\ \mathrm{H} & 0.932169 & 1.831462 & -1.846679 \\ \mathrm{H} & -2.816359 & 0.784782 & 1.055392 \\ \mathrm{H} & -1.840463 & 2.991339 & 1.150058 \\ \mathrm{H} & 0.021584 & 3.562434 & -0.366849 \\ \mathrm{C} & 0.288893 & 1.456882 & -1.052401 \\ \mathrm{C} & -1.832362 & 0.826208 & 0.583571 \\ \mathrm{C} & -1.353331 & 2.242626 & 0.537888 \\ \mathrm{C} & -0.355135 & 2.551209 & -0.279207\end{array}$

\section{TS-5b}

$\begin{array}{ll}\text { E } & -731.257685 \\ \text { H } & -731.256741 \\ \text { G } & -731.312464 \\ \text { Imag. Freq. } & -430.0855\end{array}$

Cartesian coordinates

$\begin{array}{llll}\mathrm{H} & -3.672178 & -0.157422 & -0.885123 \\ \mathrm{H} & -4.257373 & 0.15873 & 0.775905 \\ \mathrm{H} & -2.89066 & -0.953494 & 0.492538 \\ \mathrm{C} & -3.383787 & -0.03964 & 0.155827 \\ \mathrm{O} & -2.53719 & 1.083838 & 0.344592 \\ \mathrm{H} & 3.07568 & -0.193502 & -0.073244 \\ \mathrm{H} & 3.019794 & -1.864924 & 0.409999 \\ \mathrm{H} & 2.080272 & -1.442781 & -1.819306 \\ \mathrm{H} & 2.428776 & 0.01825 & 2.183616 \\ \mathrm{H} & 0.31719 & -0.483868 & 2.999964 \\ \mathrm{H} & -1.300599 & -1.882205 & 2.076202 \\ \mathrm{H} & -1.312153 & -3.008174 & 0.050474 \\ \mathrm{H} & 0.150364 & -2.735658 & -1.755054 \\ \mathrm{C} & -0.451652 & -1.689294 & 1.428675 \\ \mathrm{C} & -0.463385 & -2.355668 & 0.223034 \\ \mathrm{C} & 0.567609 & -0.882242 & 2.022597 \\ \mathrm{C} & 0.410042 & -2.205091 & -0.847304 \\ \mathrm{C} & 1.778974 & -0.566223 & 1.541493 \\ \mathrm{C} & 1.469465 & -1.276674 & -0.935705 \\ \mathrm{C} & 2.387379 & -0.984457 & 0.242692 \\ \mathrm{O} & -1.507446 & -0.311588 & -1.802534 \\ \mathrm{H} & 0.660916 & 0.037846 & -2.589765\end{array}$




$\begin{array}{llll}\mathrm{H} & -1.041004 & 2.224797 & 1.756662 \\ \mathrm{H} & 1.153368 & 2.939633 & 1.654019 \\ \mathrm{H} & 2.644346 & 2.798405 & -0.151877 \\ \mathrm{H} & 2.437875 & 1.440593 & -2.048976 \\ \mathrm{C} & 0.839523 & 2.307569 & 0.831013 \\ \mathrm{C} & 1.754689 & 2.181447 & -0.218192 \\ \mathrm{C} & -0.474474 & 1.872475 & 0.901752 \\ \mathrm{C} & 1.644232 & 1.372478 & -1.311809 \\ \mathrm{C} & -1.219094 & 1.063661 & 0.062936 \\ \mathrm{C} & 0.689601 & 0.333749 & -1.546539 \\ \mathrm{C} & -0.738711 & 0.355538 & -1.119219\end{array}$

\section{TS-S4b}

$\begin{array}{ll}\text { E } & -731.249804 \\ \text { H } & -731.248860 \\ \text { G } & -731.303978 \\ \text { Imag. Freq. } & -424.3637\end{array}$

Cartesian coordinates

$\begin{array}{lrrc}\mathrm{C} & -0.647260 & 0.260370 & 1.272540 \\ \mathrm{C} & 0.665500 & 0.426070 & 1.848750 \\ \mathrm{C} & -1.034750 & 0.763270 & -0.106800 \\ \mathrm{C} & 1.741290 & 1.170410 & 1.439330 \\ \mathrm{C} & -0.400970 & 1.927900 & -0.678720 \\ \mathrm{C} & 1.876520 & 2.001490 & 0.334520 \\ \mathrm{C} & 0.863720 & 2.415590 & -0.536370 \\ \mathrm{H} & 2.625250 & 1.081220 & 2.063050 \\ \mathrm{H} & 2.836510 & 2.490700 & 0.218270 \\ \mathrm{H} & 1.121260 & 3.234900 & -1.199740 \\ \mathrm{H} & -1.037900 & 2.424900 & -1.401190 \\ \mathrm{O} & -1.477410 & -0.402120 & 1.879250 \\ \mathrm{C} & 0.710200 & -0.390660 & -2.023110 \\ \mathrm{C} & 2.018970 & -0.530390 & -1.320210 \\ \mathrm{C} & -0.588910 & -0.652360 & -1.271630 \\ \mathrm{C} & 2.313140 & -1.284700 & -0.248040 \\ \mathrm{C} & -0.780800 & -1.854590 & -0.543410 \\ \mathrm{C} & 1.450960 & -2.122660 & 0.514200 \\ \mathrm{C} & 0.131680 & -2.471930 & 0.294240 \\ \mathrm{H} & 1.943750 & -2.663410 & 1.315190 \\ \mathrm{H} & -0.259240 & -3.265930 & 0.919350 \\ \mathrm{H} & -1.789770 & -2.245340 & -0.507560 \\ \mathrm{H} & -1.413780 & -0.452760 & -1.950920 \\ \mathrm{H} & 2.841550 & -0.004420 & -1.792610 \\ \mathrm{H} & 0.652220 & 0.626090 & -2.425240\end{array}$




$\begin{array}{rrrr}\mathrm{H} & 0.725610 & -1.043320 & -2.905220 \\ \mathrm{H} & 0.741410 & -0.091970 & 2.797150 \\ \mathrm{H} & 3.339380 & -1.249080 & 0.100670 \\ \mathrm{O} & -2.401130 & 0.961430 & -0.271710 \\ \mathrm{C} & -3.375780 & -0.060530 & -0.215750 \\ \mathrm{H} & -4.330640 & 0.462720 & -0.237310 \\ \mathrm{H} & -3.327190 & -0.705990 & -1.099950 \\ \mathrm{H} & -3.301200 & -0.656440 & 0.688550\end{array}$

TS-6b

$\begin{array}{ll}\text { E } & -731.260273 \\ \text { H } & -731.259328 \\ \text { G } & -731.313201 \\ \text { Imag. Freq. } & -81.9518\end{array}$

Cartesian coordinates

$\begin{array}{llll}\mathrm{H} & 3.706433 & 0.054462 & -0.876607 \\ \mathrm{H} & 4.249857 & -0.209715 & 0.806991 \\ \mathrm{H} & 2.916655 & 0.921464 & 0.45263 \\ \mathrm{C} & 3.393002 & -0.018066 & 0.161699 \\ \mathrm{O} & 2.514269 & -1.110199 & 0.370441 \\ \mathrm{H} & -3.100929 & 0.224237 & -0.065127 \\ \mathrm{H} & -2.882562 & 1.881259 & 0.425348 \\ \mathrm{H} & -2.074481 & 1.441192 & -1.781947 \\ \mathrm{H} & -2.434211 & -0.032894 & 2.198606 \\ \mathrm{H} & -0.284784 & 0.425543 & 3.005525 \\ \mathrm{H} & 1.351622 & 1.739967 & 2.041504 \\ \mathrm{H} & 1.259608 & 3.061735 & 0.121584 \\ \mathrm{H} & -0.221368 & 2.849991 & -1.682377 \\ \mathrm{C} & 0.477206 & 1.579718 & 1.419571 \\ \mathrm{C} & 0.453047 & 2.343938 & 0.228325 \\ \mathrm{C} & -0.534605 & 0.824369 & 2.028266 \\ \mathrm{C} & -0.39607 & 2.218891 & -0.819286 \\ \mathrm{C} & -1.753095 & 0.485597 & 1.534059 \\ \mathrm{C} & -1.411138 & 1.16877 & -0.960035 \\ \mathrm{C} & -2.339567 & 0.946205 & 0.242667 \\ \mathrm{O} & 1.560469 & 0.173714 & -1.868273 \\ \mathrm{H} & -0.606467 & 0.016312 & -2.594844 \\ \mathrm{H} & 0.919318 & -2.08710 & 1.847432 \\ \mathrm{H} & -1.320482 & -2.654141 & 1.725365 \\ \mathrm{H} & -2.631362 & -2.772013 & -0.236818 \\ \mathrm{H} & -2.352469 & -1.47064 & -2.176684 \\ \mathrm{C} & -0.941684 & -2.097293 & 0.876073 \\ \mathrm{C} & -1.786077 & -2.092389 & -0.270721\end{array}$




$\begin{array}{llll}\mathrm{C} & 0.39857 & -1.764031 & 0.952559 \\ \mathrm{C} & -1.640549 & -1.332744 & -1.369888 \\ \mathrm{C} & 1.193246 & -1.027516 & 0.096281 \\ \mathrm{C} & -0.7096 & -0.199281 & -1.533172 \\ \mathrm{C} & 0.748599 & -0.352465 & -1.124382\end{array}$

\section{TS-S5b}

$\begin{array}{lc}\text { E } & -731.252012 \\ \text { H } & -731.251068 \\ \text { G } & -731.304044 \\ \text { Imag. Freq. } & -130.2748\end{array}$

Cartesian coordinates

$\begin{array}{rrrr}\text { C } & 0.636260 & -0.232590 & 1.290820 \\ \mathrm{C} & -0.706920 & -0.297950 & 1.843620 \\ \mathrm{C} & 1.002310 & -0.682200 & -0.144080 \\ \mathrm{C} & -1.797470 & -1.011520 & 1.414420 \\ \mathrm{C} & 0.388090 & -1.954320 & -0.604980 \\ \mathrm{C} & -1.929920 & -1.821250 & 0.298900 \\ \mathrm{C} & -0.876080 & -2.389520 & -0.471100 \\ \mathrm{H} & -2.704480 & -0.862680 & 1.992160 \\ \mathrm{H} & -2.909530 & -2.258910 & 0.144660 \\ \mathrm{H} & -1.136630 & -3.282390 & -1.030260 \\ \mathrm{H} & 1.066170 & -2.537730 & -1.215820 \\ \mathrm{O} & 1.515890 & 0.284080 & 1.953720 \\ \mathrm{C} & -0.692930 & 0.296760 & -1.994590 \\ \mathrm{C} & -2.003710 & 0.438440 & -1.299590 \\ \mathrm{C} & 0.621360 & 0.497670 & -1.223600 \\ \mathrm{C} & -2.288770 & 1.261980 & -0.257440 \\ \mathrm{C} & 0.807590 & 1.829590 & -0.622750 \\ \mathrm{C} & -1.405190 & 2.051890 & 0.487050 \\ \mathrm{C} & -0.080400 & 2.459730 & 0.175590 \\ \mathrm{H} & -1.863930 & 2.600310 & 1.302720 \\ \mathrm{H} & 0.265460 & 3.347390 & 0.693370 \\ \mathrm{H} & 1.789540 & 2.275920 & -0.716300 \\ \mathrm{H} & 1.399960 & 0.339520 & -1.970320 \\ \mathrm{H} & -2.838150 & -0.049260 & -1.790160 \\ \mathrm{H} & -0.653550 & -0.692340 & -2.457900 \\ \mathrm{H} & -0.690680 & 1.006490 & -2.831310 \\ \mathrm{H} & -0.768820 & 0.210210 & 2.797830 \\ \mathrm{H} & -3.312130 & 1.247570 & 0.100750 \\ \mathrm{O} & 2.387270 & -0.953440 & -0.225280\end{array}$




$\begin{array}{lrrr}\mathrm{C} & 3.360070 & 0.067200 & -0.240640 \\ \mathrm{H} & 4.316820 & -0.448970 & -0.172550 \\ \mathrm{H} & 3.348930 & 0.626650 & -1.184190 \\ \mathrm{H} & 3.260050 & 0.749740 & 0.600050\end{array}$

\section{TS-7b \\ E $\quad-731.543543$ \\ $\mathrm{H} \quad-731.252422$ \\ G $\quad-731.306972$ \\ Imag. Freq. $\quad-259.649$}

Cartesian coordinates

$\begin{array}{lrrr}\mathrm{C} & -0.552999 & 0.365502 & -1.045491 \\ \mathrm{C} & 0.887853 & 0.130923 & -1.424635 \\ \mathrm{C} & -1.040433 & 1.259506 & -0.081882 \\ \mathrm{C} & 1.986229 & 1.080662 & -1.165697 \\ \mathrm{C} & -0.261488 & 2.138733 & 0.716420 \\ \mathrm{C} & 2.077354 & 2.013395 & -0.207843 \\ \mathrm{C} & 1.066193 & 2.416365 & 0.722212 \\ \mathrm{H} & 2.844779 & 0.961133 & -1.820219 \\ \mathrm{H} & 3.012183 & 2.560678 & -0.141383 \\ \mathrm{H} & 1.391553 & 3.118243 & 1.481749 \\ \mathrm{H} & -0.859895 & 2.642916 & 1.467207 \\ \mathrm{H} & 0.823458 & -0.013230 & -2.504137 \\ \mathrm{O} & -1.273988 & -0.499215 & -1.615170 \\ \mathrm{C} & 2.098734 & -1.431781 & 0.339181 \\ \mathrm{C} & 1.215692 & -1.387847 & -0.914215 \\ \mathrm{C} & 1.582803 & -0.761621 & 1.571722 \\ \mathrm{C} & -0.054407 & -2.131052 & -0.855919 \\ \mathrm{C} & 0.336569 & -0.862106 & 2.040343 \\ \mathrm{C} & -0.846865 & -2.296884 & 0.288685 \\ \mathrm{C} & -0.742633 & -1.637997 & 1.484636 \\ \mathrm{H} & -0.313682 & -2.730549 & -1.716761 \\ \mathrm{H} & -1.738896 & -2.897369 & 0.151519 \\ \mathrm{H} & -1.578498 & -1.780216 & 2.163007 \\ \mathrm{H} & 0.100576 & -0.337649 & 2.959352 \\ \mathrm{H} & 2.292489 & -0.171330 & 2.139149 \\ \mathrm{H} & 1.815741 & -1.811508 & -1.720755 \\ \mathrm{H} & 2.316202 & -2.481743 & 0.570601 \\ \mathrm{H} & 3.056692 & -0.978350 & 0.074001 \\ \mathrm{O} & -2.363890 & 1.448988 & 0.142548 \\ \mathrm{C} & -3.313245 & 0.412824 & -0.007031 \\ \mathrm{H} & -3.001356 & -0.476187 & 0.548939 \\ \mathrm{H} & -4.235938 & 0.809546 & 0.414855 \\ \mathrm{H} & -3.458521 & 0.135703 & -1.049068\end{array}$


TS-8b

$\begin{array}{ll}\text { E } & -731.260511 \\ \text { H } & -731.259567 \\ \text { G } & -731.310202 \\ \text { Imag. Freq. } & -658.5236\end{array}$

Cartesian coordinates

$\begin{array}{llll}\mathrm{H} & -2.341656 & -2.250351 & -1.0183 \\ \mathrm{H} & 3.075616 & -0.880244 & 0.888965 \\ \mathrm{H} & 3.320855 & -1.025306 & -0.873299 \\ \mathrm{H} & 4.324275 & 0.085512 & 0.077699 \\ \mathrm{C} & 3.324797 & -0.333776 & -0.022199 \\ \mathrm{H} & -1.741426 & -2.192225 & 1.569861 \\ \mathrm{H} & 0.019838 & -3.427179 & 0.614201 \\ \mathrm{C} & -0.121656 & -2.42519 & 0.22576 \\ \mathrm{H} & 1.457904 & -2.63159 & -1.113812 \\ \mathrm{C} & 0.670290 & -1.985672 & -0.743125 \\ \mathrm{H} & -2.752305 & 0.378063 & -1.590826 \\ \mathrm{H} & -3.301375 & -1.264117 & 0.069668 \\ \mathrm{C} & -1.926814 & -0.132825 & -1.107436 \\ \mathrm{C} & -2.302492 & -1.387021 & -0.351012 \\ \mathrm{H} & 1.312391 & -0.504226 & -2.128071 \\ \mathrm{C} & 0.629391 & -0.584773 & -1.281593 \\ \mathrm{H} & -0.668742 & 0.563742 & -2.659597 \\ \mathrm{C} & -0.689583 & 0.061142 & -1.703684 \\ \mathrm{C} & -1.277083 & -1.624304 & 0.764586 \\ \mathrm{O} & 2.459755 & 0.756568 & -0.243446 \\ \mathrm{C} & 1.089064 & 0.488901 & -0.258108 \\ \mathrm{H} & -1.01795 & -0.229141 & 2.412934 \\ \mathrm{C} & -0.899477 & -0.206034 & 1.328364 \\ \mathrm{O} & 1.286773 & 0.242011 & 2.107627 \\ \mathrm{C} & 0.561861 & 0.176373 & 1.15175 \\ \mathrm{H} & 1.043048 & 2.150335 & -1.614678 \\ \mathrm{H} & -2.869969 & 0.659063 & 1.173938 \\ \mathrm{H} & -2.397154 & 2.869401 & 0.635302 \\ \mathrm{H} & -0.554101 & 3.586427 & -0.659566 \\ \mathrm{C} & 0.424207 & 1.737362 & -0.824079 \\ \mathrm{C} & -1.865334 & 0.832906 & 0.796939 \\ \mathrm{C} & -1.589518 & 2.158323 & 0.503071 \\ \mathrm{C} & -0.507898 & 2.578455 & -0.262213\end{array}$

\section{TS-9b}




$\begin{array}{ll}\text { E } & -731.273361 \\ \text { H } & -731.272417 \\ \text { G } & -731.323397 \\ \text { Imag. Freq. } & -596.6542\end{array}$

Cartesian coordinates

$\begin{array}{llll}\mathrm{H} & -4.058167 & 0.757859 & -0.728295 \\ \mathrm{H} & -3.112424 & 0.388869 & 0.732517 \\ \mathrm{H} & -3.663174 & -0.936753 & -0.316794 \\ \mathrm{C} & -3.310699 & 0.092454 & -0.300514 \\ \mathrm{O} & -2.152855 & 0.255794 & -1.098959 \\ \mathrm{H} & 2.846609 & -0.116031 & 1.249277 \\ \mathrm{H} & 2.141884 & 1.421047 & 1.712908 \\ \mathrm{H} & 0.921718 & -0.363311 & 2.648416 \\ \mathrm{H} & 2.972482 & 1.402638 & -0.585695 \\ \mathrm{H} & 1.137646 & 2.538635 & -1.624922 \\ \mathrm{H} & -0.420866 & 3.556741 & -0.210642 \\ \mathrm{H} & -1.560341 & 2.480569 & 1.547685 \\ \mathrm{H} & -1.299001 & 0.261361 & 2.190926 \\ \mathrm{C} & -0.019078 & 2.604651 & 0.116600 \\ \mathrm{C} & -0.688849 & 1.968370 & 1.152584 \\ \mathrm{C} & 0.88635 & 1.970053 & -0.732345 \\ \mathrm{C} & -0.52801 & 0.649435 & 1.528486 \\ \mathrm{C} & 1.989017 & 0.978418 & -0.365398 \\ \mathrm{C} & 0.736499 & -0.179524 & 1.585673 \\ \mathrm{C} & 1.987897 & 0.539019 & 1.087139 \\ \mathrm{O} & -1.752171 & -2.255941 & 0.205504 \\ \mathrm{H} & 0.18424 & -2.279947 & 1.742364 \\ \mathrm{H} & -0.232779 & 0.663022 & -2.389655 \\ \mathrm{H} & 2.005249 & 0.104743 & -2.332428 \\ \mathrm{H} & 2.73759 & -2.001844 & -1.58942 \\ \mathrm{H} & 1.849852 & -3.158834 & 0.311605 \\ \mathrm{C} & 1.575255 & -0.129679 & -1.358454 \\ \mathrm{C} & 1.971062 & -1.527214 & -0.987203 \\ \mathrm{C} & 0.105873 & 0.291796 & -1.429856 \\ \mathrm{C} & 1.492847 & -2.164659 & 0.072308 \\ \mathrm{C} & -0.958583 & -0.205867 & -0.667061 \\ \mathrm{C} & 0.424187 & -1.573118 & 0.949966 \\ \mathrm{C} & -0.865488 & -1.433735 & 0.146377\end{array}$




\section{Endo Parent System Transition States}

\section{Cyclic TS}

$\begin{array}{lr}\mathrm{E} & -465.565468 \\ \mathrm{H} & -465.329974 \\ \mathrm{G} & -465.375884 \\ \text { Imag. Freq. } & -534.493\end{array}$

Cartesian coordinates

$\begin{array}{lrrr}\mathrm{C} & -0.637254 & -0.993202 & 1.536009 \\ \mathrm{C} & -0.745870 & -1.468768 & 0.089173 \\ \mathrm{C} & -0.413908 & 0.475861 & 1.734280 \\ \mathrm{C} & -1.800593 & -1.057631 & -0.736460 \\ \mathrm{C} & -0.860859 & 1.456929 & 0.941611 \\ \mathrm{C} & -2.167857 & 0.268065 & -0.906232 \\ \mathrm{C} & -1.614838 & 1.368502 & -0.278283 \\ \mathrm{H} & -2.185682 & -1.774445 & -1.453691 \\ \mathrm{H} & -2.860824 & 0.481855 & -1.713813 \\ \mathrm{H} & -1.911495 & 2.330259 & -0.684279 \\ \mathrm{H} & -0.594983 & 2.468612 & 1.231254 \\ \mathrm{H} & 0.151450 & 0.757109 & 2.615595 \\ \mathrm{H} & -0.528930 & -2.531658 & 0.024320 \\ \mathrm{C} & 2.065850 & -0.968695 & 0.053133 \\ \mathrm{H} & 2.887078 & -1.461972 & -0.480418 \\ \mathrm{H} & 1.906062 & -1.542435 & 0.965772 \\ \mathrm{C} & 2.414059 & 0.462138 & 0.283328 \\ \mathrm{H} & 3.103923 & 0.803651 & 1.041049 \\ \mathrm{C} & 1.769040 & 1.231522 & -0.619990 \\ \mathrm{H} & 1.874074 & 2.302627 & -0.721570 \\ \mathrm{C} & 0.882288 & -0.894528 & -0.904673 \\ \mathrm{H} & 0.770613 & -1.692110 & -1.626072 \\ \mathrm{C} & 0.851819 & 0.441161 & -1.366864 \\ \mathrm{H} & 0.309058 & 0.781344 & -2.233426 \\ \mathrm{H} & -1.545471 & -1.312952 & 2.060651 \\ \mathrm{H} & 0.173859 & -1.538013 & 2.024436\end{array}$

\section{Acyclic TS}

$\begin{array}{lr}\mathrm{E} & -389.357014 \\ \mathrm{H} & -389.137063 \\ \mathrm{G} & -389.184035 \\ \text { Imag. Freq. } & -554.303\end{array}$

Cartesian coordinates 


$\begin{array}{lrrc}\mathrm{C} & 2.376326 & 0.176613 & 0.729661 \\ \mathrm{C} & -2.533663 & -1.386951 & 0.380423 \\ \mathrm{C} & 2.308370 & -0.982927 & -0.032780 \\ \mathrm{C} & -1.379321 & -1.707365 & -0.215860 \\ \mathrm{C} & 1.100802 & -1.624625 & -0.307694 \\ \mathrm{C} & -0.101387 & -1.224991 & 0.222467 \\ \mathrm{H} & 3.179917 & -1.272595 & -0.608974 \\ \mathrm{H} & 1.092140 & -2.393692 & -1.074472 \\ \mathrm{H} & -0.112663 & -0.594484 & 1.101658 \\ \mathrm{H} & -1.394324 & -2.330632 & -1.106154 \\ \mathrm{H} & -3.483635 & -1.740914 & 0.001409 \\ \mathrm{H} & 3.350343 & 0.627285 & 0.882368 \\ \mathrm{C} & -2.256848 & 1.670120 & -0.109779 \\ \mathrm{H} & -3.032927 & 2.057658 & 0.536876 \\ \mathrm{C} & -0.960948 & 1.786137 & 0.207258 \\ \mathrm{H} & -0.692526 & 2.269637 & 1.144640 \\ \mathrm{C} & 1.458170 & 1.611505 & -0.334098 \\ \mathrm{H} & 2.156362 & 1.601004 & -1.159430 \\ \mathrm{C} & 0.126288 & 1.317710 & -0.603678 \\ \mathrm{H} & -0.130576 & 0.846937 & -1.544156 \\ \mathrm{H} & 1.653478 & 2.387238 & 0.399770 \\ \mathrm{H} & -2.569292 & 1.218963 & -1.042984 \\ \mathrm{H} & 1.709475 & 0.298166 & 1.573836 \\ \mathrm{H} & -2.552509 & -0.785917 & 1.280092\end{array}$




\section{References}

(1) Bannwarth, C.; Ehlert, S.; Grimme, S. GFN2-XTB-An Accurate and Broadly Parametrized Self-Consistent Tight-Binding Quantum Chemical Method with Multipole Electrostatics and Density-Dependent Dispersion Contributions. J. Chem. Theory Comput. 2019, 15 (3), 1652-1671. https://doi.org/10.1021/acs.jctc.8b01176.

(2) Grimme, S.; Bannwarth, C.; Dohm, S.; Hansen, A.; Pisarek, J.; Pracht, P.; Seibert, J.; Neese, F. Fully Automated Quantum-Chemistry-Based Computation of Spin-Spin-Coupled Nuclear Magnetic Resonance Spectra. Angew. Chem. Int. Ed. Engl. 2017, 56 (46), 1476314769. https://doi.org/10.1002/anie.201708266.

(3) Chai, J.-D.; Head-Gordon, M. Long-Range Corrected Hybrid Density Functionals with Damped Atom-Atom Dispersion Corrections. Phys. Chem. Chem. Phys. 2008, 10 (44), 6615-6620. https://doi.org/10.1039/B810189B.

(4) Schäfer, A.; Huber, C.; Ahlrichs, R. Fully Optimized Contracted Gaussian Basis Sets of Triple Zeta Valence Quality for Atoms Li to Kr. J. Chem. Phys. 1994, 100 (8), 5829-5835. https://doi.org/10.1063/1.467146.

(5) Weigend, F.; Ahlrichs, R. Balanced Basis Sets of Split Valence, Triple Zeta Valence and Quadruple Zeta Valence Quality for H to Rn: Design and Assessment of Accuracy. Phys. Chem. Chem. Phys. 2005, 7 (18), 3297-3305. https://doi.org/10.1039/B508541A.

(6) Frisch, M. J.; Trucks, G. W.; Schlegel, H. B.; Scuseria, G. E.; Robb, M. A.; Cheeseman, J. R.; Scalmani, G.; Barone, V.; Petersson, G. A.; Nakatsuji, H.; et al. Gaussian 16 Revision A.03. 2016.

(7) Linder, M.; Brinck, T. On the Method-Dependence of Transition State Asynchronicity in Diels-Alder Reactions. Phys. Chem. Chem. Phys. 2013, 15 (14), 5108-5114. https://doi.org/10.1039/C3CP44319A.

(8) Mardirossian, N.; Head-Gordon, M. Thirty Years of Density Functional Theory in Computational Chemistry: An Overview and Extensive Assessment of 200 Density Functionals. Mol. Phys. 2017, $115 \quad$ (19), 2315-2372. https://doi.org/10.1080/00268976.2017.1333644.

(9) Xue, X. S.; Jamieson, C. S.; Garcia-Borràs, M.; Dong, X.; Yang, Z.; Houk, K. N. Ambimodal Trispericyclic Transition State and Dynamic Control of Periselectivity. J. Am. Chem. Soc. 2019, 141 (3), 1217-1221. https://doi.org/10.1021/jacs.8b12674.

(10) Neese, F. The ORCA Program System. Wiley Interdiscip. Rev. Comput. Mol. Sci. 2012, 2 (1), 73-78. https://doi.org/10.1002/wcms.81.

(11) Neese, F. Software Update: The ORCA Program System, Version 4.0. WIREs Comput. Mol. Sci. 2018, 8 (1), e1327. https://doi.org/10.1002/wcms.1327.

(12) Guo, Y.; Riplinger, C.; Becker, U.; Liakos, D. G.; Minenkov, Y.; Cavallo, L.; Neese, F. Communication: An Improved Linear Scaling Perturbative Triples Correction for the Domain Based Local Pair-Natural Orbital Based Singles and Doubles Coupled Cluster Method [DLPNO-CCSD(T)]. J. Chem. Phys. 2018, 148 (1), 11101. https://doi.org/10.1063/1.5011798.

(13) Dunning, T. H. Gaussian Basis Sets for Use in Correlated Molecular Calculations. I. The Atoms Boron through Neon and Hydrogen. J. Chem. Phys. 1989, 90 (2), 1007-1023. https://doi.org/10.1063/1.456153.

(14) Weigend, F.; Köhn, A.; Hättig, C. Efficient Use of the Correlation Consistent Basis Sets in Resolution of the Identity MP2 Calculations. J. Chem. Phys. 2002, 116 (8), 3175-3183. 
https://doi.org/10.1063/1.1445115.

(15) Weigend, F. Accurate Coulomb-Fitting Basis Sets for H to Rn. Phys. Chem. Chem. Phys. 2006, 8 (9), 1057-1065. https://doi.org/10.1039/B515623H.

(16) Luchini, G.; Alegre-Requena, J.; IFunes; Rodríguez-Guerra, J.; Chen, J.; Paton, R. Bobbypaton/GoodVibes: $\quad$ GoodVibes $\quad$ v3.0.0. https://doi.org/10.5281/ZENODO.3346166.

(17) Alecu, I. M.; Zheng, J.; Zhao, Y.; Truhlar, D. G. Computational Thermochemistry: Scale Factor Databases and Scale Factors for Vibrational Frequencies Obtained from Electronic Model Chemistries. J. Chem. Theory Comput. 2010, 6 (9), 2872-2887. https://doi.org/10.1021/ct100326h.

(18) Li, Y.-P.; Gomes, J.; Mallikarjun Sharada, S.; Bell, A. T.; Head-Gordon, M. Improved Force-Field Parameters for QM/MM Simulations of the Energies of Adsorption for Molecules in Zeolites and a Free Rotor Correction to the Rigid Rotor Harmonic Oscillator Model for Adsorption Enthalpies. J. Phys. Chem. C 2015, 119 (4), 1840-1850. https://doi.org/10.1021/jp509921r.

(19) Grimme, S. Supramolecular Binding Thermodynamics by Dispersion-Corrected Density Functional Theory. Chem. $\quad-\quad A \quad$ Eur. J. 2012, 18 (32), 9955-9964. https://doi.org/10.1002/chem.201200497. 